\title{
Determining Reliable Networks of Prepositioning Materiel Warehouses for Public-Sector Rapid Response Supplies
}

\author{
Thomas E. Lang ${ }^{1}$ and Ronald G. McGarvey ${ }^{2}$ \\ ${ }^{1}$ LMI, 7940 Jones Branch Drive, Tysons, VA 22102, USA \\ ${ }^{2}$ Department of Industrial and Manufacturing Systems Engineering and Harry S. Truman School of Public Affairs, \\ University of Missouri, E3437 Lafferre Hall, Columbia, MO 65211, USA \\ Correspondence should be addressed to Ronald G. McGarvey; mcgarveyr@missouri.edu
}

Received 23 May 2016; Revised 9 August 2016; Accepted 22 August 2016

Academic Editor: Konstantina Skouri

Copyright (c) 2016 T. E. Lang and R. G. McGarvey. This is an open access article distributed under the Creative Commons Attribution License, which permits unrestricted use, distribution, and reproduction in any medium, provided the original work is properly cited.

\begin{abstract}
Events such as natural disasters or combat operations require a rapid response capability from humanitarian service providers and military organizations. Such organizations can decrease their response times through the prepositioning of materiel in forward warehouses, reducing the time needed to transport items to the site of need. A particular challenge to the development of networks of prepositioning warehouses is that the warehouses themselves may be impacted by the very disruptions that drive demands for prepositioned materials. The objective of this research is to identify a reliable network posture, which is a set of utilized facility locations and an allocation of materiel to those locations, that can satisfy time-sensitive delivery requirements to potential locations around the globe, ensuring that demands can be satisfied even in the event of loss of access to a subset of storage sites (along with said sites' materiel), all at minimum total cost. We develop new optimization formulations to account for differing levels of network reliability, all reflecting the time-sensitive environment faced by rapid response operations. We demonstrate an application of this methodology using rapid response material prepositioned by the US Air Force.
\end{abstract}

\section{Introduction}

Events such as natural disasters or combat operations require a rapid response capability from humanitarian service providers and military organizations. Such organizations can decrease their response times through the prepositioning of materiel in forward warehouses, reducing the time needed to transport items to the site of need. For example, the US Air Force (USAF) maintains a global combat support network of prepositioning sites to allow it to rapidly commence operations at remote locations. These warehouses store items such as tents, electricity generators, construction vehicles, and medical supplies that are difficult to transport quickly over long distances or difficult to be procured in remote locations (or both). In its simplest form, a prepositioning network is a set of demand nodes (forward operating locations [FOLs] from which forward-deployed forces operate), supply nodes (forward supply locations [FSLs] from which support resources are sourced and sent to FOLs), and network links connecting the two node sets.

In the absence of uncertainty, this problem reduces to the well-known multicommodity location-allocation and transportation problem. Many researchers have examined the impact of "demand-side" uncertainty, in which the set of future demands is not perfectly known, on such problems, typically utilizing approaches such as stochastic programming or robust optimization. A related, but distinct, aspect of uncertainty is the potential for unplanned disruptions to the network itself; it is this "supply-side" uncertainty that is of concern to this paper.

(1) Network Risk. All types of networks run the risk of disruptions when parts of the system fail. These failures may occur along an arc (e.g., an obstacle along a transportation route), or at a node itself. The duration of the disruption can vary between temporary and long-term. The magnitude 
of the disruption also can vary between a temporary effect on a small portion of the network and causing the entire network to fail. In this research, we will focus on node failures, that is, failures occurring at FSLs. Node failure can occur for many reasons; for military applications it might take the form of denial of access by host countries, such as when Kyrgyzstan announced that it would be closing the US air base at Manas [1], or the deliberate targeting of warehouses by an adversary; for humanitarian response it might occur due to natural disasters such as a tsunami that not only impacts the residents of littoral areas, but also destroys all warehouses located near commercial ports. Since it is not possible for such a rapid responder to obtain ensured access from every potential storage location, their networks of prepositioning sites should be constructed in such a manner as to limit the impact of node disruption on the network's response times.

While the terms "robustness" and "reliability" are often used interchangeably, several authors [2, 3] have used these terms to distinguish two different concepts. Under this terminology, supply chain robustness refers to the extent to which a supply network is able to handle an uncertain future demand, which can be viewed as a demand-side issue. In contrast, supply chain reliability refers to the ability of a system to perform well even when parts of the system fail. Reliability models hedge against uncertainty in the network itself, which can be viewed as a supply-side issue.

In addition to the definitional difference, the robustness and reliability of a network must be evaluated differently. To a large extent, sensitivity analysis can usually evaluate the robustness of a system; however, evaluating the reliability of a system requires more advanced modeling techniques [2-4].

Network reliability models are concerned with the chances of a network remaining connected even in the face of random failures. Such optimization models seek to maximize the probability that the networks will remain connected. These models appear in areas such as telecommunications and computer networks $[5,6]$. Traditionally, such studies have focused on failures occurring along the network routes and not at the nodes.

Backup covering models focus on assigning customers to multiple facilities with each facility serving a prespecified fraction of demand. Optimization models within this class seek to maximize expected coverage when facility availability is uncertain, using the concept of primary and secondary node assignments. Much of the work done in this area has looked at where to locate emergency service vehicles and socioeconomic applications [7-10].

Supply chain disruption models provide techniques for designing and operating supply chains that are resilient to disruptions of all sorts. These disruptions may occur along network routes or at the nodes. Optimization models within this class seek to minimize the expected cost of initial network design as well as transportation and ongoing operational costs. The majority of the research on supply chain disruption models has been qualitative.

A quantitative examination of supply chain disruption was provided by Cui et al. [11], who examined reliable facility location under potential disruptions. In this study, the authors assumed access might be lost to each potential facility according to a site-dependent probability and developed a solution procedure that would minimize the sum of the normal operating cost and the expected failure cost. In the introduction to this paper, the authors noted a fundamental difference between their research and the problem type studied in this paper:

\section{Realistically, no company would accept a supply network with high normal operating costs just to hedge against very rare facility disruptions.}

Observe that, for prepositioning applications, the demand to be served is either of a humanitarian nature, in which case the supplies are intended to prevent loss of life, or of a military nature, in which case national sovereignty is viewed to be potentially at risk. In either of these cases, the cost of a delayed response is so great that a rapid response must be guaranteed across the entire range of considered scenarios, and the idea of site-dependent failure probabilities does not apply.

Commercial applications of reliable facility location design were extended by Shen et al. [12], who considered an application in which either a failure probability is assumed to be known for each potential facility or a probability of occurrence is assumed for a set of scenarios defining which facilities are operational. These authors attempt to minimize the facility opening cost plus the expected transportation and penalty costs, considering only incapacitated facility, and provide a general nonlinear integer programming formulation, along with a special formulation for the case of uniform failure probabilities. A recent review of operations research models for supply chain disruptions was provided by Snyder et al. [13].

Morton et al. [14] addressed the vulnerability of military sealifts to attacks. They began by using deterministic mixedinteger programming models to provide an assignment and routing of ships to deliver cargo as efficiently as possible. To account for the fact that an enemy may disrupt the deployment by attacking the network in some forward node, the authors used a stochastic-programming model.

In 1995, Mulvey et al. [15] conducted work on distribution-free robust optimization (RO) of large-scale systems, observing that many "mathematical programming models are assumed to be deterministic and are typically formulated by solving a worst-case scenario." Snyder et al. [16] also looked at RO. The authors created an RO model which optimized across multiple-scenario sets rather than within a single-scenario set. The work addressed the following question: how should spending be distributed over programs to achieve the maximum, balanced capabilities across these programs under a number of possible futures? Like Mulvey et al. [15], the authors also address the idea of models which solve a worst-case scenario. They note that it can be tempting to think that solutions which satisfy the worst-case scenario will also prepare better for other, less challenging scenarios. This is not usually the case as the proportions of resources needed for each scenario set are generally different. In other words, the worst-case scenario may require a different mix of resources than less demanding scenarios. 
Finally, fortification models attempt to make a supply network more resilient to attacks by identifying the weak components to the network so that resources can be concentrated on fortifying these components. In a 2004 study, Salmeron et al. [17] analyzed the security and resilience of electrical power grids against disruptions caused by terrorist attacks. Their approach began by identifying the set of attacks that causes the largest possible disruptions. Their model, as a result, "identifies grid components (nodes) which, when "hardened," yield the best improvement in system security." A weakness of their approach is that actual terrorist "resources" are uncertain so the model solutions are highly dependent upon these assumptions. In a 2006 study, Brown et al. [18] take a more general approach, the bilevel programming model, using an attacker-defender (and therefore maxmin) type method. The key assumption is that the attacker has a perfect model of how the defender will optimally operate his system, and the attacker will manipulate that system to the best of its advantage. Every node is considered vulnerable to attack unless it has been hardened. The model is able to determine the value of protecting network components, hardening them, or of adding new components into the system for purposes of redundancy. Amouzegar et al. [19] and McGarvey et al. [20] performed initial examinations of such robustness and reliability concepts with regard to the USAF combat support network.

One paper which considers a similar problem is the 2013 study by Galindo and Batta [21], which examines prepositioning of hurricane relief supplies. However, these authors do not consider the time-phasing of delivery of supplies, nor do they consider a multiregional system in which supplies can be transported from one area to another. Instead, this study focuses on stocking more than the expected demand for supplies in a single region, under the assumption that an upper bound is known for the percentage of prepositioned supplies that might be destroyed by a hurricane.

Our paper differs from the prior research examining network disruptions on multicommodity location-allocation and transportation problems due to the lack of recourse available to the logistics system. Observe that, for rapid response systems, the potential disruptions to the prepositioning network will occur at exactly the time of the demands on the network (whether due to a natural disaster that impacts both the population requiring assistance and the nearby logistics sites or an adversary who coordinates his attacks on both the logistics system and targets of military and political significance). Moreover, when the duration of potential network disruptions exceeds the allowable response windows (which are typically very short for rapid response capabilities), it is not possible either to produce new assets quickly enough or to move assets from disrupted facilities to other staging sites-the network must have redundant capabilities of sufficient size on hand to compensate for the potential losses. Note also that stochastic models, utilizing a known probability distribution specifying the likelihood of disruption, are not generally applicable to this problem, since attacks from an adversary are not random, but would instead be selected to maximize damage to the logistics system. This paper presents an approach for identifying minimum-cost approaches to building such a level of network reliability into a multicommodity location-allocation and transportation network.

\section{Mathematical Model}

The following section presents a mixed-integer linear programming (MILP) model that determines an optimal set of FSLs to open and an allocation of prepositioning materiel resources to each opened FSL, in order to satisfy the timephased demands for rapid response materiel at FOLs, subject to storage and transportation constraints, even in the event of loss of access to a subset of the opened FSLs. When establishing a logistics support network, policymakers can generate network reliability through combinations of the following options, all of which are captured in our model:

(i) Opening additional FSLs.

(ii) Increasing the inventory of prepositioned stocks.

(iii) Dispersing commodities more evenly across FSLs.

This MILP formulation will identify a minimum-cost solution that satisfies all constraints, rather than including cost considerations in the form of a budget constraint. We elect to use such a formulation because this analysis is bestsuited for supporting what is referred to as programming decisions. During programming, the capabilities called for in planning guidance are translated into resource requirements. Subsequent analysis prioritizes expenditures across programs in order to satisfy fiscal guidance requirements and identifies the impact of not fully funding individual programs. The solutions returned by this model identify the full funding requirements for the desired capability: the delivery of rapid response WRM materiel to a set of FOLs within time-phased requirements, even in the event of loss of access to WRM storage sites.

Define the following sets:

I: commodity index

$J$ : FSL index

$K$ : FOL index

$M$ : transportation mode index

$T$ : time index

The optimization model makes use of the following data elements:

$\bar{\omega}_{j k m}$ : number of time periods necessary to load a mode $m$ vehicle at FSL $j$, the transit time from FSL $j$ to FOL $k$, the number of time periods necessary to unload the vehicle at FOL $k$, and any additional time needed after unloading to transport the commodities

$D_{i k t}$ : cumulative demand, in tons, for commodity $i$ at FOL $k$ by time $t$

$E_{j}$ : minimum units of storage needed for economically feasible FSL at location $j$

$F_{j}$ : maximum potential units of storage space at FSL $j$ 
$\mathrm{IA}_{i j}$ : initial allocation of commodity $i$ at FSL $j$

$\gamma_{m}$ : maximum load in tons per mode $m$ vehicle

$\sigma_{m}$ : utilization rate, expressed (for airlift) as the average flying hour goal per day divided by 24 hours, for mode $m$

$C_{m}$ : initial number of transportation vehicles available

$\tau_{j k m}$ : one-way transportation time from FSL $j$ to FOL

$k$ (in either direction) via mode $m$

$A_{j m}$ : MOG capacity for mode $m$ vehicles at FSL $j$

$B_{k m}$ : MOG capacity for mode $m$ vehicles at FOL $k$

$\alpha_{m}$ : number of time periods necessary to load a mode $m$ vehicle

$\beta_{m}$ : number of time periods necessary to unload a mode $m$ vehicle

$\zeta_{k}$ : contingency start period at FOL $k$

$\eta_{k}$ : contingency finish period at FOL $k$

$\phi_{i}$ : conversion factor for commodity $i$ from tons to square feet of storage space

$\Delta_{j}$ : fixed cost incurred to open FSL $j$ with $E_{j}$ square feet of storage space

$\Xi_{j}$ : construction cost per unit of storage needed beyond $E_{j}$ at FSL $j$

$\Upsilon_{j}$ : operating cost (discounted over the time horizon) per unit of storage at FSL $j$

$\Omega_{i j k m}$ : cost per ton of commodity $i$ transported from FSL $j$ to FOL $k$ via mode $m$

$\Theta_{m}$ : cost of obtaining an additional vehicle of mode $m$

$P_{i}$ : cost of procuring additional tons of commodity $i$

$L_{i}$ : cost of reallocating tons of commodity $i$ (this cost is assumed to be constant, independent of which FSLs are reallocating or receiving the commodity)

$\Psi_{i k}$ : shortfall cost per time unit per ton of commodity $i$ not fulfilled at FOL $k$

The optimization model's decision variables are defined as follows (all variables nonnegative):

$w_{j}$ : binary variable indicating status of FSL $j, w_{j}=1$ when FSL is open and $w_{j}=0$ otherwise $x_{i j k m t}$ : quantity (tons) of commodity $i$ sent from FSL $j$ to FOL $k$ via mode $m$ at time $t$

$y_{j k m t}$ : number of mode $m$ vehicles tasked to transport commodities from FSL $j$ to FOL $k$, beginning loading on time $t$, integer variable

$s_{i k t}$ : shortfall below demand for commodity $i$ at FOL $k$ not fulfilled by time $t$ (note that although the model allows for shortfall to be tracked, all of the computation experience presented in this paper will assume that no shortfall is allowed; i.e., $s_{i k t}=$ $0 \forall i, k, t)$

$n n_{j}$ : additional units of storage (square feet) needed beyond $E_{j}$ at FSL $j$

$\mathrm{AU}_{i j}$ : additional units of commodity $i$ that is procured at FSL $j$ (this is a gain of inventory at FSL $j$ )

$\mathrm{EI}_{i j}$ : inventory of commodity $i$ at FSL $j$ that is reallocated to other FSLs (this is a loss of inventory at FSL $j$ )

$\mathrm{AP}_{i}$ : sum of the additional amount of commodity $i$ procured across the entire system

$q_{j m}$ : number of mode $m$ vehicles available at FSL $j$ at start of initial time period $t=1$

$v_{j m t}$ : number of mode $m$ vehicles available at FSL $j$ at the end of time $t$

$z_{j k m t}$ : number of mode $m$ vehicles tasked to make the return trip from FOL $k$ to FSL $j$, departing on time $t$

$r_{m}$ : additional number of mode $m$ transportation vehicles procured

$w w_{j}$ : units of storage utilized at FSL $j$ (we distinguish between utilized and nonutilized storage space because operating and maintenance costs will differ between the two)

$u_{i k}$ : maximum amount of commodity $i$ sent to FOL $k$ from any single FSL

2.1. Nonreliable Model. To begin, we first present an MILP formulation that does not account for potential loss of access to FSLs. We then present three variations on this model that incorporate network reliability. The initial model, referred to hereafter as the nonreliability model (NRM), is defined by objective (1) and constraints (2) through (15) below:

$$
\begin{array}{ll}
\min & \sum_{j}\left(\Delta_{j} w_{j}+\Xi_{j} n n_{j}+\Upsilon_{j} w w_{j}\right)+\sum_{i j k m t} \Omega_{i j k m} x_{i j k m t}+\sum_{m} \Theta_{m} r_{m}+\sum_{i} P_{i} \mathrm{AP}_{i}+\sum_{i j} L_{i} \mathrm{EI}_{i j}+\sum_{i k t} \Psi_{i k} s_{i k t}, \\
& \sum_{j m, n \leq t} x_{i j k m\left(n-\bar{\omega}_{j k m}\right)} \geq D_{i k t}-s_{i k t} \quad \forall i, k, t, \\
& \sum_{i k m t} \phi_{i} x_{i j k m t} \leq E_{j} w_{j}+n n_{j} \quad \forall j, \\
& n n_{j} \leq\left(F_{j}-E_{j}\right) w_{j} \quad \forall j,
\end{array}
$$




$$
\begin{aligned}
& \sum_{k m t} x_{i j k m t}+\mathrm{EI}_{i j} \leq \mathrm{IA}_{i j}+\mathrm{AU}_{i j} \quad \forall i, j \\
& \sum_{j} \mathrm{AU}_{i j} \leq \sum_{j} \mathrm{EI}_{i j}+\mathrm{AP}_{i} \quad \forall i \\
& \sum_{i} x_{i j k m t} \leq \gamma_{m} y_{j k m t} \quad \forall j, k, m, t \\
& \sum_{j} q_{j m} \leq\left(C_{m}+r_{m}\right) \quad \forall m, \\
& \sum_{k} y_{j k m t} \leq v_{j m(t-1)} \quad \forall j, m ; t \geq 2, \\
& v_{j m " 1 "}=q_{j m}-\sum_{k} y_{j k m " 1 "} \quad \forall j, m, \\
& v_{j m t}=v_{j m(t-1)}+\sum_{k}\left[z_{j k m\left(t-\tau_{j k m}\right)}-y_{j k m t}\right] \quad \forall j, m ; t \geq 2 \text {, } \\
& \sum_{k} \sum_{n=0}^{\alpha_{m}-1} y_{j k m(t-n)} \leq A_{j m} \quad \forall j, t, m, \\
& \sum_{j} \sum_{n=0}^{\beta_{m}-1}\left(y_{j k m\left(t-\tau_{j k m}-\alpha_{m}-n\right)}\right) \leq B_{k m} \quad \forall k ; \zeta_{k} \leq t \leq \eta_{k}, \\
& \sum_{j} z_{j k m t}=\sum_{j} y_{j k m\left(t-\bar{\omega}_{j k m}\right)} \quad \forall k, m, t \\
& \sum_{j k t}\left[\tau_{j k m}\left(y_{j k m t}+z_{j k m t}\right)\right] \leq\left(C_{m}+r_{m}\right) \sigma_{m} \quad \forall m, \\
& \sum_{i k m t} \phi_{i} x_{i j k m t} \leq w w_{j} \quad \forall j .
\end{aligned}
$$

Equation (1) is our objective function. Demand constraint (2) requires cumulative arrivals for commodity $i$ at FOL $k$ by time $t$ to satisfy the cumulative demand by time $t$. Constraints (3) and (4) limit the space available for storage of commodities at FSL $j$ and also control the opening of FSLs. Constraints (5) and (6) control the allocation of commodities across FSLs, including potential reallocations of commodities away from existing FSLs. Constraint (7) limits the total weight of commodities that can be shipped on each individual mode $m$ vehicle. Constraint (8) imposes limits on the total number of vehicles available system-wide. Flow balance equations (9), (10), and (11) track the available vehicles at each FSL $j$ across all time periods $t$.

FSL MOG constraints are defined in such a way as to account for both the amount of physical space the vehicle takes up on the ground and the amount of time the vehicle is on the ground. The MOG at each FSL is modeled separately for each of the three classes of vehicles. Air, ground, and sea vehicles each use different loading equipment and also have different physical space requirements (e.g., water ports versus airstrips). Each FSL is assumed to have a maximum number of vehicle spaces allowed for loading each class of vehicle at any one time. Within each class of vehicle, different modes are assumed to consume different fractions of this loading space. Also, each of these different modes of transport is assumed to consume the loading space for different lengths of time. Constraints (12) are the MOG constraints at each FSL $j$ at each time period $t$, defined over all modes $m$ within each vehicle class. A total of three FSL MOG constraints exist for each FSL $j$ at each time period $t$, one for each vehicle class (air, ground, and sea). FOL maximum-on-ground constraints (13) similarly restrict the FOLs based on the available unload space at each FOL. Once vehicles $y_{j k m t}$ finish unloading at FOL $k$, constraint (14) reassigns those vehicles to return trips to FSLs. Notice that this model formulation does not assign an individual transport vehicle to a single FSL, to a single FOL, or to a single commodity type. Instead, for example, a given aircraft may transport commodities from FSL $j_{1}$ to FOL $k_{1}$. After unloading at FOL $k_{1}$, the aircraft may then make the return trip to FSL $j_{2}$ where it may be loaded with a different commodity type. Further, individual FOLs do not necessarily have all of their demand shipped from one FSL. Instead, multiple FSLs may send commodities to a given FOL. Constraint (15) limits the average fleetwide utilization for each transport mode over the entire scenario duration, although in practice we will only apply this constraint to 
aircraft. Utilization rate is expressed as the average flying hour goal per day divided by 24 hours. Finally, constraint (16) ensures that the amount of storage space utilized at an FSL is sufficient to hold all of the commodities that will be sourced from that FSL.

The model is solved by finding the set of decision variable values that satisfies the set of contingency requirements and also minimizes the costs of conducting training and deterrent exercises over a given time horizon. The model outputs a transportation plan and reports the time needed for FOLs to receive all required commodities.

2.2. Initial Reliability Model. If a policymaker knew in advance when and where FSL access would be lost, the NRM could be used to make decisions to fortify the network against this loss. In reality, the location and timing of future disruptions are unknown, and the policymaker needs to design a network which can satisfy demands, regardless of loss of access to any utilized FSL(s). We next discuss the changes made to the NRM to construct a reliability model (RM) that identifies a single posture (set of FSLs and allocation of commodities across FSLs). The majority of the variables and constraints from the NRM can be utilized in the RM; accordingly, we will present here only the model components that are changed in the RM. A detailed presentation of the mathematical formulation of the RM appears in Appendix.

In order to construct the RM, we need to identify which decisions are and are not subject to recourse. Nonrecourse refers to a variable or constraint for which a single decision is made that cannot be changed as loss-of-access scenarios occur and thus must be applied across all potential loss-ofaccess scenarios. Construction is an example of a nonrecourse decision. Once a decision is made on the number and size of facilities needed, it takes time to build these facilities. When loss of FSL access occurs, there is no sufficient time to construct new facilities to compensate for the lost FSL.

In contrast, as loss of FSL access occurs, transportation decisions can be made in real-time and are thus considered a recourse decision. An important example of a constraint allowing for recourse is the demand constraint. The demand constraint requires that the quantity of commodity $i$ sent from FSL $j$ to FOL $k$ be greater than or equal to the cumulative demand of commodity $i$ at FOL $k$ by time $t$. Since this clearly depends on the availability of FSL $j$, we will allow for recourse. In the RM, recourse will be modeled by modifying the definition of FSL set $J$, creating $\|J\|$ new subsets. Given a set of potential FSLs with $\aleph=|J|$, we define new sets, $J_{1}$ through $J_{\aleph}$. Each of these new sets is a subset of the original $J$, each containing $\mathrm{N}-1$ FSLs, with a different FSL removed from each. For example, subset $J_{1}$ contains all of the FSLs except for FSL $j_{1}$.

In the RM, we replace variables $x_{i j k m t}$ and $s_{i k t}$ with the following definitions (italic text here emphasizes the differences with respect to the previously defined variables):

$x 1_{i j k m t}$ : quantity of commodity $i$ sent from FSL $j \in J_{1}$ to FOL $k$ via mode $m$, beginning loading on time $t$, allowing the model to identify valid movements in the event of loss of access to FSL $j_{1}$; we create $\aleph-1$ similar variables corresponding to loss of access to each potential FSL

$s 1_{i k t}:$ shortfall below demand for commodity $i$ at FOL $k$ not fulfilled by time $t$ when access is lost to FSL $j_{1}$; we create $N-1$ similar variables corresponding to loss of access to each potential FSL

This formulation thus requires creation of $N$ versions of each variable; that is, we need to create variables $\left(x 1_{j k m t}\right.$, $x 2_{j k m t}, \ldots, x \aleph_{j k m t}$ ) to account for a potential loss of access at each FSL.

In a similar manner, the model allows for recourse in the demand constraints, generating $\aleph+1$ versions of the demand constraint: one for the full set of FSLs $(J)$ and one for each subset of FSLs $\left(J_{1}, \ldots,\right)$. The constraint corresponding to the full set FSLs is identical to constraint (2) above from the NRM. For each of the other $\aleph$ demand constraints, we ensure that demand can also be satisfied when commodities cannot be shipped from each individual FSL (i.e., in the event that access is lost to each potential FSL). The new demand constraints are defined as

$$
\begin{gathered}
\sum_{m, n \leq t, j \in J_{1}} x 1_{i j k m\left(n-\bar{\omega}_{j k m}\right)} \geq D_{i k t}-s 1_{i k t} \quad \forall i, k, t, \\
\vdots \\
\sum_{m, n \leq t, j \in J_{\mathbb{N}}} x \aleph_{i j k m\left(n-\bar{\omega}_{j k m}\right)} \geq D_{i k t}-s \aleph_{i k t} \quad \forall i, k, t .
\end{gathered}
$$

We make similar modifications to the variables related to vehicle tracking $\left(y_{j k m t}, v_{j m t}, z_{j k m t}\right)$, creating $\aleph$ new versions of each variable in the same manner as for variables $\left(x 1_{j k m t}, x 2_{j k m t}, \ldots\right)$. In a manner similar to that employed in (17) above, we need to create $\aleph$ additional versions of each constraint for constraints (7), (9), (10), (11), (12), (13), (14), and (15).

The RM needs to identify the amount of each commodity to allocate to each FSL, which is a decision that does not allow for recourse; a decision is made once at the outset regarding how much of each commodity to place at each FSL. To do this, the RM utilizes a new variable:

$x x_{i j}$ : maximum total tons of commodity $i$ sent from FSL $j$, across the full set $(J)$ of FSLs, and all $\aleph$ subsets of FSLs $\left(J_{1}, \ldots, J_{\aleph}\right)$.

Constraints (18) below ensure that no more than $x x_{i j}$ total tons of commodity $I$ are sourced from FSL $j$, across the complete set of FSLs and all $\aleph$ subsets of FSLs:

$$
\begin{gathered}
\sum_{k m t} x_{i j k m t} \leq x x_{i j} \quad \forall i, j, \\
\sum_{k m t} x 1_{i j k m t} \leq x x_{i j} \quad \forall i ; j \in J_{1}, \\
\vdots \\
\sum_{k m t} x \aleph_{i j k m t} \leq x x_{i j} \quad \forall i ; j \in J_{\aleph} .
\end{gathered}
$$


FSL storage capacity is a nonrecourse constraint, appearing in the NRM as constraint (3) and modified in the RM through use of constraint (19), in which the left-hand side term makes use of the $x x_{i j}$ variable:

$$
\sum_{i} \phi_{i} x x_{i j} \leq E_{j} w_{j}+n n_{j} \quad \forall j
$$

A similar modification was made to the constraint controlling for the total amount of commodities in the entire system, appearing in the NRM as constraint (5), modified in the RM through use of constraint (20):

$$
x x_{i j}+\mathrm{EI}_{i j} \leq \mathrm{IA}_{i j}+\mathrm{AU}_{i j} \quad \forall i, j
$$

Constraints (4), (6), and (8) remain in the RM unchanged from their formulation in the NRM.

The objective function also needs to be modified in the RM to account for the new variable definitions. The RM objective function formulation minimizes the average cost across all $\aleph+1$ FSL loss scenarios considered (alternative objectives such as minimizing the worst-case cost under any FSL loss scenario could also be accommodated with a slightly different objective formulation; we did not consider such objectives in this study):

$$
\begin{aligned}
\min & \sum_{j}\left(\Delta_{j} w_{j}+\Xi_{j} n n_{j}+\Upsilon_{j} w w_{j}\right)+\left[\sum_{i j k m t} \frac{\Omega_{i j k m}}{\aleph+1} x_{i j k m t}+\sum_{i k m t} \sum_{j \in J_{1}} \frac{\Omega_{i j k m}}{\aleph+1} x 1_{i j k m t}+\cdots+\sum_{i k m t} \sum_{j \in J_{\aleph}} \frac{\Omega_{i j k m}}{\aleph+1} x \aleph_{i j k m t}\right] \\
& +\sum_{m} \Theta_{m} r_{m}+\sum_{i} P_{i} \mathrm{AP}_{i}+\sum_{i j} L_{i} \mathrm{EI}_{i j}+\left[\sum_{i k t} \frac{\Psi_{i k}}{\aleph+1} s_{i k t}+\sum_{i k t} \frac{\Psi_{i k}}{\aleph+1} s 1_{i k t}+\cdots+\sum_{i k t} \frac{\Psi_{i k}}{\aleph+1} s \aleph_{i k t}\right] .
\end{aligned}
$$

2.3. Alternative Reliability Model. Although the RM presented in the previous section identifies a single reliable posture which satisfied all constraints even when access was lost to any individual FSL within the posture, the approach utilized here cannot be extended to examine the case of simultaneous loss of access to multiple facilities, because the number of FSL subsets grows rapidly (from $\aleph$ subsets for potential loss of access to any single potential FSL, to $\left(\begin{array}{c}\mathrm{N} \\ 2\end{array}\right)$ subsets for potential loss of access to any pair of potential FSLs). In this section, we present the mathematical details of an alternative reliability model (ARM), which, at the expense of poorer objective function performance, can be expanded to consider simultaneous loss of access to multiple facilities.

We will first present the ARM considering loss of access to a single potential FSL (denote this model as ARM-1), before expanding to the multiple FSL failure case. We initially return to the NRM formulation. The same basic sets of variables and constraints remain in the ARM as done in the NRM. We discuss here only the constraints and variables that are modified in the ARM; all variables and constraints not discussed below remain unchanged from the NRM.

We first introduce a new data element:

$D_{i k}^{\prime}=\sum_{t} D_{i k t} \forall i, k$ : total demand for commodity $i$ at FOL $k$ over all time periods

Two NRM constraints are modified in the ARM:

$$
\begin{gathered}
\sum_{m t} x_{i j k m t} \leq u_{i k} \quad \forall i, j, k \\
\sum_{j m, n \leq t} x_{i j k m\left(n-\bar{\omega}_{j k m}\right)} \geq D_{i k t}\left(1+\frac{u_{i k}}{D_{i k}^{\prime}}\right)-s_{i k t} \quad \forall i, k, t .
\end{gathered}
$$

Constraint (22) constrains the total amount of commodity $i$ sent from FSL $j$ to FOL $k$ shipped via all modes of transportation over all time periods to be less than or equal to the variable $u_{i k}$. Demand constraint (23) requires cumulative arrivals for commodity $i$ at FOL $k$ by time $t$ to satisfy the cumulative demand by time $t$. Further, in addition to satisfying cumulative demand, cumulative arrivals must also satisfy an additional amount of demand equal to the maximum amount of commodity $i$ sent to FOL $k$ from any FSL. This demand constraint ensures that if access is lost to any individual FSL and that FSL was the maximum supplier of commodity $i$ to FOL $k$, the FOL will still receive enough commodity $i$ to satisfy demand from the remaining FSLs in the combat support network. Observe that if the FSL to which access was lost is not the maximum supplier of commodity $i$ to some FOL $k$, then that FOL could elect to not utilize the most-expensive shipments in excess of its requirement.

With minor modifications, the ARM can be used to examine the impact of multiple, simultaneous facility losses. A model that allows all demands to be met even in the event of simultaneous loss of access to any two potential FSLs (denoted as ARM-2) can be constructed by redefining the variable $u_{i k}$ and replacing constraint (22) with constraint (24) below:

$u_{i k}$ : maximum amount of commodity $i$ sent to FOL $k$ from any two FSLs

$$
\sum_{m t}\left(x_{i j_{1} k m t}+x_{i j_{2} k m t}\right) \leq u_{i k} \quad \forall i, j_{1}, j_{2}, k ; j_{1} \neq j_{2} \text {. }
$$

Previously $u_{i k}$ variable was set equal to the maximum amount drawn from any single facility. We now redefine $u_{i k}$ to be the maximum amount drawn from any pair of facilities. Observe that the demand constraint now ensures that if access is lost to any two FSLs simultaneously and that those FSLs were the two largest suppliers of commodity $i$ 


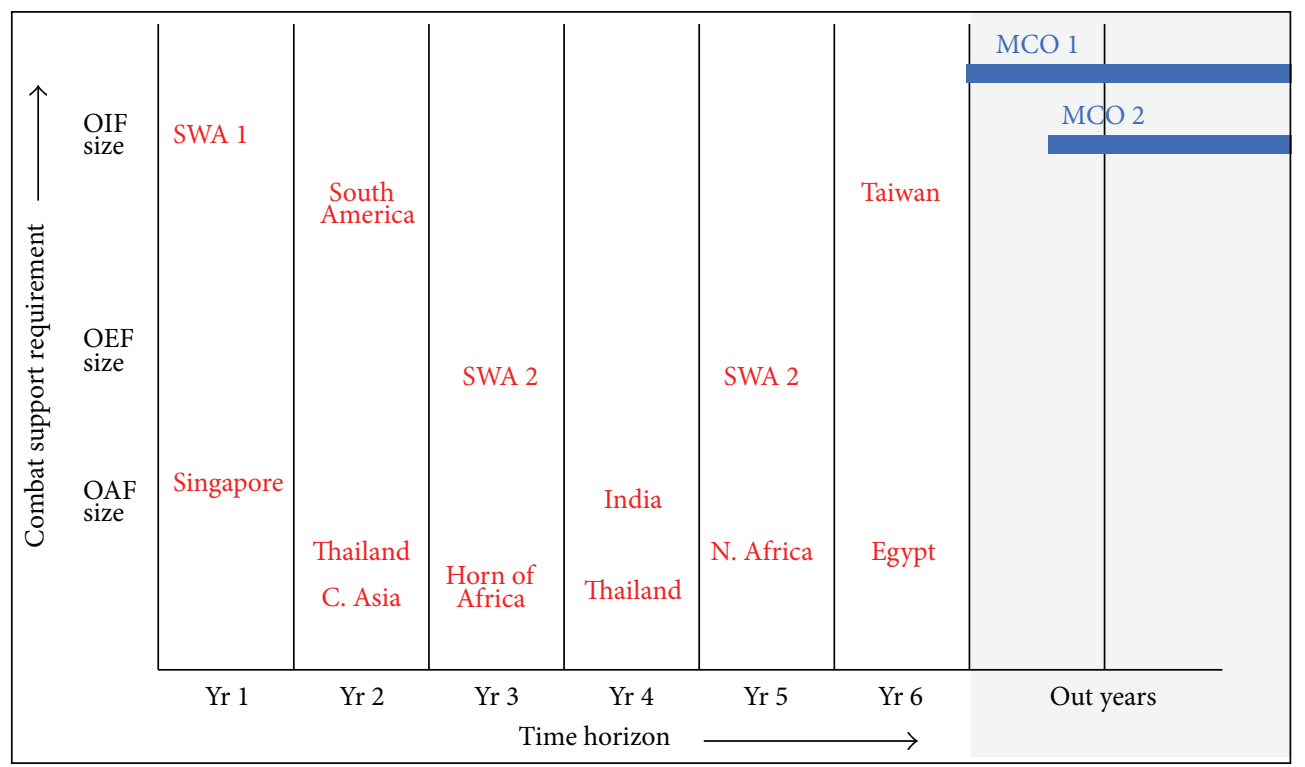

(MCO) Major Combat Operation (OAF) Operation Allied Force

(OIF) Operation Iraqi Freedom (OEF) Operation Enduring Freedom

FIGURE 1: Evaluated scenario, with combat support requirements over time.

to FOL $k$, the FOL will still receive enough commodity $i$ to satisfy demand from the remaining FSLs in the combat support network. While we will not demonstrate it in this paper, the model can easily be expanded to account for three or more simultaneous facility failures. To identify a posture that can meet all demands in the event of loss of access to $n$ facilities, one would redefine $u_{i k}$ variable as the maximum amount of commodity $i$ sent to FOL $k$ from any $n$ FSLs.

\section{Application}

In the following section, we illustrate this modeling approach with an application to a military rapid response scenario. Our methodology begins with the identification of scenarios to be supported. Each scenario is a series of time-phased deployments, occurring in various locations around the world, requiring differing levels of combat support requirements at the FOLs. These requirements, combined with the set of potential FSLs and FOLs that are derived from the scenarios, serve as first sets of inputs for the models to follow.

3.1. Scenario Construction. The unpredictability of the future security environment requires the evaluation of support concepts across a broad range of combat and noncombat scenarios with varying degrees of intensity, across a wide range of geographical areas. These scenarios include strategic factors such as deployment distance, likely amount of strategic warning, and deployment duration. The scenarios have varying degrees of infrastructure richness, such as availability of fuel, communications, and transportation. The engagements can be classified into three categories: Major Combat Operations (MCOs), exercises and other deterrent missions, and humanitarian and military operations other than warfare (MOOTW).

Given a set of scenarios, we next computed the required number of aircraft by type and location, also known as the Air Order of Battle, using historical data and expert judgment. Given an Air Order of Battle for each scenario, we estimated the combat support requirements needed at each FOL using the model of Snyder and Mills [22]. Although combat support is comprised of many consumable and reparable items, we focus here on items known as war reserve materiel (WRM) that are prepositioned in global warehouses; common WRM items include billeting, munitions, and vehicles.

With the deployments defined, we can now outline the sequencing and recurrence of those deployments. We chose to schedule the deployments and contingencies into a scenario comprising a six-year time frame to align with the Planning, Programming, Budgeting, and Execution (PPBE) system's future years defense program (FYDP). Two MCOs are included in order to sufficiently size the facilities to support regional conflicts specified in the planning guidance. We are assuming that non-MCOs would be halted in the event of an MCO. This allows us to model the MCOs at the end of the six-year time horizon without the loss of fidelity.

The scenario, presented in Figure 1, focuses on supporting a number of deployments in the Persian Gulf region, Asian Littoral, South America, and Africa over a time horizon of six years. We have scheduled the MCOs in the scenario for execution at the end of the six-year period. This approach focuses attention on providing resources to support deterrent 
deployments to ensure their funding while also placing $\mathrm{MCO}$ requirements in the Planning, Programming, Budgeting, and Execution (PPBE) process.

The vertical axis in Figure 1 illustrates the variability of demands across the considered engagements, in terms of the aggregate combat support requirements (measured here in tons of materiel). The relative scale of recent deployments is noted alongside the vertical axis to allow for comparison. Observe that, in certain instances, deterrence operations (e.g., South America) require greater combat support than a traditional major regional conflict such as Operation Allied Force (OAF). This is due to the fact that the FOLs considered in OAF are primarily well-developed locations with significant existing infrastructure, while the South American contingency requires deployment to more austere locations. Therefore, although the OAF deployment may be much larger in terms of forces deployed, its requirements on WRM are less than those of the "smaller" deployment. In each region there may be several deployments, exercises, or deterrent missions, each with its own unique logistical characteristics.

3.2. Other Data Elements. There are a number of remaining data elements needed to define a scenario. These elements include vehicle alternatives and their characteristics, airfield throughput capacity, and candidate FSL sites.

We consider three classes of vehicles in the model: air, ground, and sea. Allowing for alternative modes of transportation might bring some FSLs into the solution set that otherwise may have been deemed infeasible or too costly. Vehicles are defined by their capacity (tons and square feet), loading and unloading time, a space conversion factor, and a utilization [23] rate. For this analysis, we consider one vehicle type per class: C-17 (air vehicle), 20-ton capacity truck (land vehicle), and Joint High Speed Vessel (sea vehicle).

An important data element that shows the capacity of an airfield to receive aircraft is the maximum-on-ground (MOG) capability. MOG generally refers to the maximum number of parking spaces an airfield can provide (parking MOG), but it can be specialized to include the maximum number of aircraft that can be served by maintenance, aerial port, or other facilities (working MOG). In our analysis, we used both parking MOG and working MOG to compute an overall measure of airfield capability. Although this discussion has focused on air vehicles, similar throughput factors apply to all modes of transportation.

We generated an initial list of over 300 potential FSL sites worldwide. Vulnerability, physical constraints, and political restrictions were taken into consideration in an attempt to reduce this list to a more manageable size. We also examined the potential for storing combat support resources aboard an afloat preposition ship (APS). Although afloat prepositioning does offer additional flexibility and reduced vulnerability versus land-based storage, the APS is much more expensive than land-based storage and presents a serious risk with regard to deployment time. APS storage is relatively expensive, since the ship is continuously operating and always generating operating costs, even when there is no demand for the items stored on board. Moreover, APS storage does not necessarily
TABLe 1: Potential FSLs.

\begin{tabular}{|c|c|}
\hline Location & Country \\
\hline Bagram & Afghanistan \\
\hline Darwin & Australia \\
\hline Baku & Azerbaijan \\
\hline Shaikh Isa & Bahrain \\
\hline Burgas & Bulgaria \\
\hline Djibouti Ambouli & Djibouti \\
\hline Cotopaxi & Ecuador \\
\hline Beni Suef & Egypt \\
\hline Spangdahlem AB & Germany \\
\hline Souda Bay & Greece \\
\hline Andersen AFB & Guam \\
\hline Chennai & India \\
\hline Chhatrapati Shivaji & India \\
\hline Balad & Iraq \\
\hline Aviano AB & Italy \\
\hline Kadena AB & Japan \\
\hline Bishkek-Manas & Kyrgyzstan \\
\hline Kaduna & Nigeria \\
\hline Masirah & Oman \\
\hline Seeb & Oman \\
\hline Thumrait & Oman \\
\hline Masroor & Pakistan \\
\hline Tocumen & Panama \\
\hline Clark & Philippines \\
\hline Okecie & Poland \\
\hline Roosevelt Roads & Puerto Rico \\
\hline Al Udeid AB & Qatar \\
\hline Sao Tome & Sao Tome \\
\hline Dakar & Senegal \\
\hline Paya Lebar & Singapore \\
\hline Louis Botha & South Africa \\
\hline Moron $\mathrm{AB}$ & Spain \\
\hline U Tapao & Thailand \\
\hline Incirlik $A B$ & Turkey \\
\hline Diego Garcia & United Kingdom \\
\hline Lakenheath & United Kingdom \\
\hline Eielson AFB & United States \\
\hline APS & Guam (home port) \\
\hline
\end{tabular}

allow for rapid delivery of items, since APS ships are typically relatively large and slow cargo ships. Even if a generous advance warning is assumed to allow for steaming toward a scenario's geographic region, it can be difficult to find a port that is capable of handling these large cargo ships and offloading the cargoes in a timely manner. Ultimately, we settled on the 38 potential sites (including an APS) presented in Table 1.

3.3. Computational Results. Each of the four models (NRM, RM, ARM-1, and ARM-2) was implemented in the General Algebraic Modeling System (GAMS) software version 23.0.2 
TABLE 2: Computational comparison of the four models.

\begin{tabular}{lccc}
\hline Model & Variables & Constraints & $\begin{array}{c}\text { Computational } \\
\text { run time }(\mathrm{s})\end{array}$ \\
\hline NRM & $\sim 80,000$ & $\sim 46,000$ & 168 \\
RM & $\sim 1,000,000$ & $\sim 570,000$ & 4200 \\
ARM-1 & $\sim 80,000$ & $\sim 52,000$ & 200 \\
ARM-2 & $\sim 80,000$ & $\sim 159,000$ & 1600 \\
\hline
\end{tabular}

and solved using CPLEX version 11.1.1 on a computer with an Intel Core 2 Duo T6600 (2.20 GHz/800 Mhz FSB/2 MB cache). Table 2 presents the problem size and computational run time associated with each model for the scenario developed in Section 2. The modeled set of scenarios includes 5 commodity types (set $I$ ), 38 candidate FSLs (set $J$ ), 40 FOLs (set $K)$, 3 transportation modes (set $M$ ), and a maximum of 10 days for delivery (set $T$ ) although some scenarios have fewer than 10 days available for delivery.

As discussed earlier, the computing resource needed to solve the RM grows exponentially versus that required for the NRM, with 920,000 new variables and 524,000 new constraints (an increase of greater than $1200 \%$ over the NRM in each case) to solve a model that can accommodate a single FSL failure. The ARM reduces the size of the problem so that it consumes much less computing power, allowing us to potentially solve problems addressing loss of access to multiple FSLs. The primary tradeoff is that while the ARM identifies a solution that is truly feasible in the event of FSL failure, it does not necessarily identify a minimum-cost solution, as discussed in the previous section.

The solution to each of the four models generates an optimal posture, which is a set of FSLs to be utilized and an allocation of resources across the utilized FSLs. Figure 2 is a map showing the FSLs utilized in each model solution. Figure 3 presents the total tons of commodities (aggregated across all commodity types) stored at each FSL for each model solution.

We evaluated the reliability of the NRM posture by fixing its set of utilized FSLs and commodity allocations to FSLs and then performing ten new model runs, each time denying access to a different one of the utilized FSLs. In all cases, the model returned an infeasible solution-it is not able to satisfy the time-phased demands from the remaining set of FSLs.

Due to the size of the RM, it was necessary to use an iterative process to obtain a solution. We first solved a relaxation of the RM, relaxing all integer variables, and identified which $w_{j}$ variables had a nonzero value in the optimal solution. Using only these twelve FSLs, we then reran the RM problem, now also relaxing the integrality of the vehicle variables, $y_{j k m t}$ and $z_{j k m t}$. This solution returned an objective value within one percent of the original (full integer restrictions) RM's root relaxation solution. This posture provides location $\left(w_{j}\right)$ and allocation $\left(x x_{i j}\right)$ variable values (i.e., nonrecourse decisions) that determine the RM posture. We evaluated the validity of this RM solution by running its posture through the NRM. We fixed the RM posture of utilized FSLs and commodity allocations to FSLs and ran the NRM (applying all integer restrictions) 12 times, each time forcing a different FSL out of the solution set; the model returned a feasible solution in every case.

We used a similar procedure to evaluate the validity of the ARM-1 posture. For this posture, we fixed its set of utilized FSLs and commodity allocations to FSLs and ran the NRM (applying all integer restrictions) 17 times, each time forcing a different FSL out of the solution set. The model returned a feasible solution in every case, ensuring that the ARM-1 posture could indeed satisfy all demands even in the event of loss of access to any single FSL.

When we initially solved the ARM-2, the model was determined to be infeasible. This was due to the constraint on the total number of vehicles available system-wide, which is insufficient to move the total amount of commodities when the movements from lost-access FSLs are included in the calculations (note that these movements would not actually occur, since access was lost to the FSLs). In all model runs, we assumed that 5000 ground vehicles, 50 air vehicles, and 4 sea vehicles were available worldwide at any one time to transport WRM. Note that these values are much less than the total number of assets actually potentially available (in particular, because many of the vehicles used for this transportation can be leased from commercial vendors). We increased the total number of transport vehicles in constraint (4.7) by a "vehicle multiplier" of 1.60 (minimum value necessary to allow for a feasible solution) to identify a set of utilized FSLs and allocation of commodities across those FSLs; note, again, that these additional vehicles would not actually be used, since the corresponding movements would not actually occur. We then evaluate the validity of this posture in a similar manner to that employed previously. We fixed the ARM-2 posture's set of utilized FSLs and commodity allocations to FSLs and ran the NRM (without using the vehicle multiplier mentioned above) over 20 times, each time forcing a different pair of FSLs out of the solution set. Note that we did not perform a validation run for all 153 possible combinations, instead focusing on those FSLs with the largest allocations of each commodity type. The model returned a feasible solution in every case, ensuring that the ARM-2 posture could satisfy all demands in the event of simultaneous loss of access to any two facilities, with one exception. When we forced the combination of the Afloat Prepositioning Ship (APS) and Cotopaxi, Ecuador, closed, then there are no remaining FSLs allowing for trucking of assets to certain South American FOLs. In this instance, the model returns an infeasible solution unless the number of aircraft in the system is increased, in line with the vehicle multiplier mentioned above. For this one instance, we included the multiplier, and the model returned a feasible solution.

3.3.1. Contrasting Cost across the Four Postures. In order to compare the costs of the solutions obtained from these four different model runs, we first evaluate each posture (set of utilized FSLs and commodity allocations to FSLs) by running it through the NRM, to obtain the total system cost in the event of no loss of access, as presented in Figure 4. 


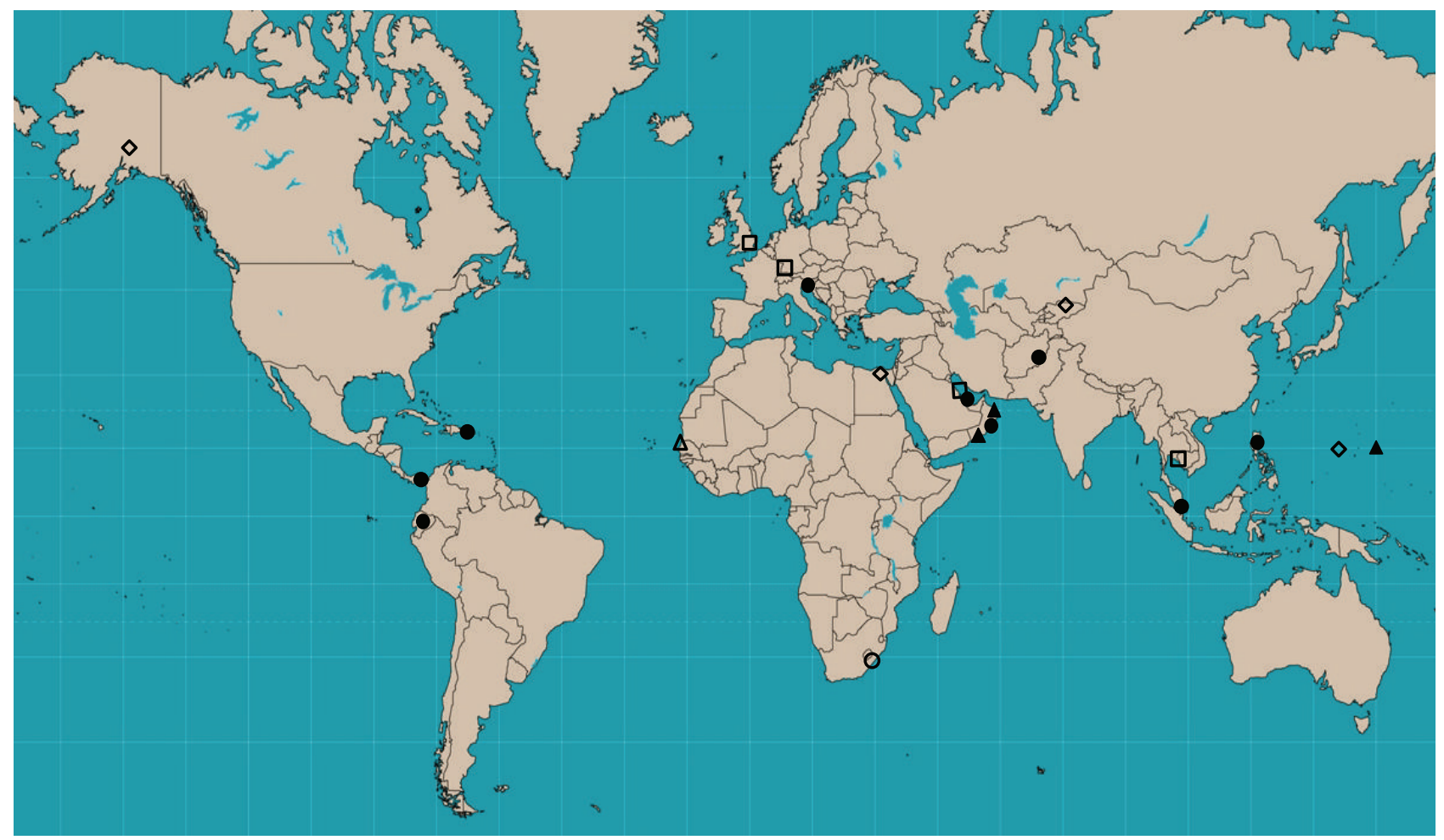
All four models
NRM \& ARM-2
ARM-1 only

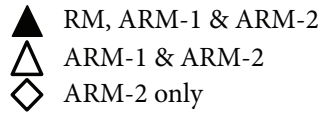

FIGURE 2: Utilized FSLs, across all model runs.
The total costs of the NRM posture under this scenario were approximately $\$ 88$ million with construction costs totaling \$26 million, transportation costs of \$20 million, and operations and maintenance $(\mathrm{O} \& \mathrm{M})$ costs of $\$ 42$ million (all costs amortized over ten years). The total costs of the RM posture under this scenario were approximately \$269 million, a 208\% increase over the NRM cost. Note that a significant portion of this cost is due to the $\$ 118$ million cost associated with the lease and operations of the APS ship based out of Andersen AFB, accounting for $65 \%$ of the difference in cost between the NRM and RM postures. Excluding the APS cost, the remaining cost difference between the two runs is approximately $\$ 63$ million. This cost is almost entirely attributable to increased procurement for the RM posture, as discussed above.

Considering next the cost of the ARM-1 posture under this scenario, its total cost of approximately $\$ 330$ million is a $23 \%$ increase over the RM cost, for a posture that offers no guarantee of increased reliability above that of the RM posture. The primary difference between the ARM-1 cost and the RM cost is a $\$ 60$ million increase in procurement cost for the ARM-1, again consistent with the inventory requirement discussion appearing above. Of course, increasing the overall system inventory increases not only procurement costs, but also O\&M costs, although this increase ( $\$ 5$ million versus the RM solution) is much more modest.
Finally, we observe that the total cost of the ARM-2 posture under this scenario is approximately $\$ 387$ million, a $44 \%$ increase over the cost of the RM posture. Again, most of this cost increase is due to a $\$ 114$ million increase in procurement versus that of the RM posture. Interestingly, the ARM-2 posture has the smallest transportation costs of the three reliable postures (approximately $\$ 23$ million, versus $\$ 31$ million and $\$ 30$ million for the RM and ARM-1, resp.), since the larger number of FSLs in the ARM-2 posture covers a broader geographic region, increasing the likelihood that an FOL will receive commodities from an FSL in the same region (this effect is particularly pronounced for the FOLs in Africa). However, the posture with the smallest total transportation costs is the NRM (approximately \$20 million), since this posture is placing a very large number of assets in a small number of particularly cost-effective FSLs.

The construction costs between the three reliable model runs do not vary significantly, despite the fact that each reliable posture uses a different number of FSLs. This is because the construction costs vary across FSLs, primarily due to differences in existing infrastructure across FSLs, but also due to differences in construction costs across different nations. The reliable postures with greater numbers of utilized FSLs generally add capability at sites with existing infrastructure (e.g., Andersen AB, Guam, or RAF Lakenheath, UK). 


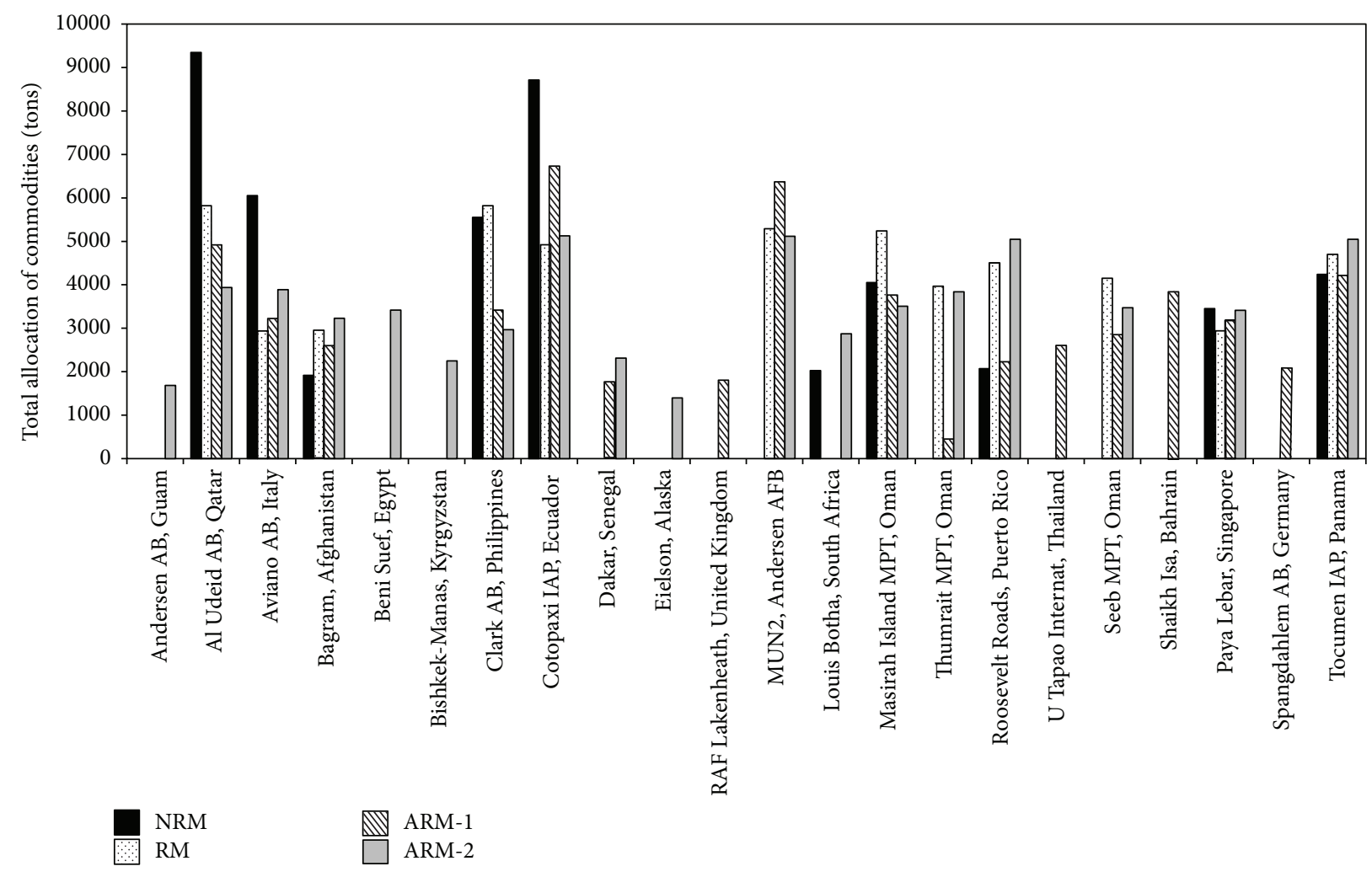

FIgURE 3: Allocation of commodities to FSLs, across all model runs.

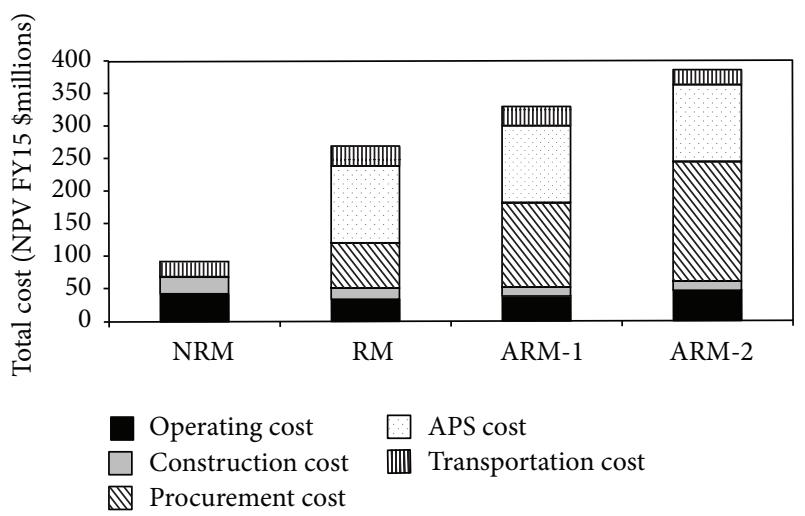

FIgURE 4: Costs, assuming no loss of access occurs.

Observe also that APS storage is utilized for all three reliable postures. In fact, for the set of scenarios considered, APS storage is required to obtain a feasible solution, since all of the reliable models are infeasible if APS storage is not allowed. This suggests that, despite its relatively high cost and its limited delivery speed, APS storage is a costeffective part of a network strategy for ensuring that networks of rapid response materiel can satisfy time-phased delivery requirements even in the event of loss of access to storage sites.

However, the scenario in which we assume no loss of access to any FSLs is not the primary scenario of interest to

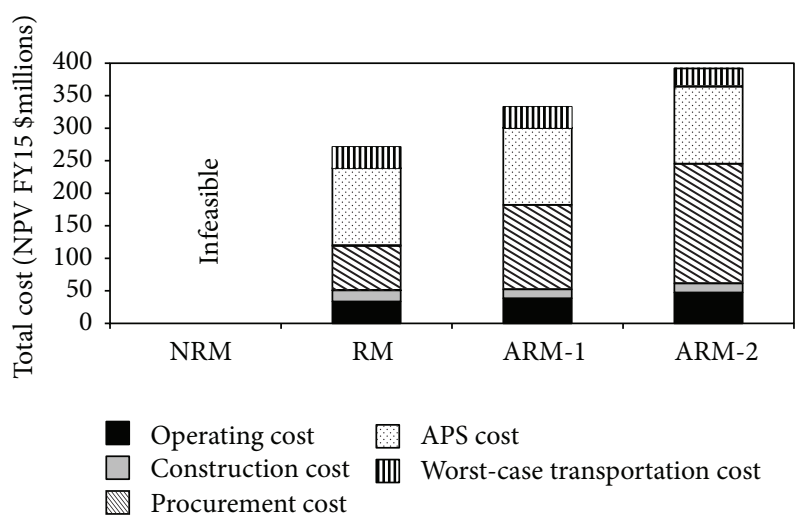

FIgURE 5: Costs, assuming loss of access to a single FSL.

this study. The motivation of this analysis was to identify postures that can satisfy the time-phased demands for delivery of assets even in the event of loss of access to FSLs. Accordingly, we evaluated the performance of each posture in the event that access was lost to a single FSL; these costs are presented in Figure 5.

As discussed above, the NRM posture is incapable of satisfying all demands if access is lost to any of its 10 FSLs. Each of the three reliable postures is capable of satisfying all demands, even if access is lost to any single FSL; that is, each is feasible with partial recourse (to transportation decisions only) across the loss of access scenarios considered. For each 
TABLE 3: Summary of model results.

\begin{tabular}{lccccccc}
\hline Model & \# of FSLs opened & Total materiel procured (tons) & Transportation & APS & $\begin{array}{c}\text { Costs }(\sim \$ M) \\
\text { Procurement }\end{array}$ & Construction & Operating \\
\hline NRM & 10 & - & $\$ 20$ & $\$ 0$ & $\$ 0$ & $\$ 26$ & $\$ 42$ \\
RM & 12 & 5,818 & $\$ 31$ & $\$ 118$ & $\$ 70$ & $\$ 17$ & $\$ 34$ \\
ARM-1 & 17 & 8,609 & $\$ 30$ & $\$ 118$ & $\$ 130$ & $\$ 14$ & $\$ 39$ \\
ARM-2 & 18 & 15,099 & $\$ 23$ & $\$ 118$ & $\$ 184$ & $\$ 14$ & $\$ 48$ \\
\hline
\end{tabular}

of the RM, ARM-1, and ARM-2 postures, we ran the posture through the NRM, removing access to a single different FSL in each run, and identified the cost under each loss-of-access scenario. Note that most of the cost components are not impacted by the occurrence of loss of access to an FSL; thus, there is no change to the operating cost, construction cost, procurement cost, or APS cost for any of the reliable postures in Figure 5, when compared to the costs presented in Figure 4 for the scenario assuming no loss of access. The only cost component that would change in the event of loss of access to an FSL is the transportation cost associated with moving assets to FOLs in support of the demands. For each reliable posture, Figure 5 presents the transportation cost for the loss of FSL access run that generated the largest (worst-case) transportation cost. These transportation cost increases are very small relative to the transportation costs for the no loss of access scenario, with total increases of $\$ 2$ million, $\$ 2.6$ million, and $\$ 4.2$ million for the RM, ARM-1, and ARM2 , respectively. Performing a similar analysis for the ARM2 posture under loss of access to any two FSLs, we observed a transportation cost increase of $\$ 6.3$ million over the cost presented in Figure 5. Table 3 summarizes the results of the four models.

3.3.2. Options for Generating Network Reliability. Policymakers can generate network reliability through combinations of the following three options: opening additional FSLs, increasing the inventory of prepositioned stocks, and dispersing commodities more evenly across FSLs. We next compare the extent to which the postures identified by each model made use of each of these options.

(1) Option 1: Opening Additional FSLs. As might be expected, the NRM posture, with ten FSLs, utilizes the fewest storage locations. The RM posture utilizes 12 FSLs, the ARM-1 posture utilizes 17 FSLs, and the ARM-2 posture utilizes 18 FSLs. Observe that nine FSLs appear in all four model solutions, three FSLs appear in every robust model solution (i.e., all solutions except NRM), two FSLs appear in two model solutions, and eight FSLs appear in a single model solution. In all postures, the FSLs are dispersed geographically, with no single region containing a majority of the facilities.

All of the FSLs opened by the NRM were also opened by the RM with the exception of Louis Botha, South Africa. No FSLs were opened in Africa for the RM run; the RM satisfies demand requirements in Africa primarily from FSLs in the Middle East. Considering the three FSLs that were opened by the RM but not opened by the NRM, the two facilities in Oman (Seeb and Thumrait) already have an extensive USAF warehousing presence, generating a significant cost advantage for these sites. The other site selected by the RM, but not the NRM, suggests the importance of a presence in South America. Since the FSL at Cotopaxi, Ecuador, supports the majority of operations in the area for the NRM, if access may be lost to Cotopaxi, the RM chooses to utilize afloat storage with the APS, to cover potential requirements in this region.

Considering next the locations selected by ARM-1, this posture utilizes all 12 FSLs utilized by the RM. The ARM-1 posture makes increased use of European FSLs, with sites at Lakenheath, UK, and Spangdahlem, Germany. The ARM1 posture also utilizes new FSLs in Africa (Dakar, Senegal), Southwest Asia (Shaikh Isa, Bahrain), and Southeast Asia (U Tapao, Thailand). The ARM-2 posture again utilizes all 12 FSLs utilized by the RM and shares African FSLs with the NRM posture (Louis Botha, South Africa) and the ARM-1 posture (Dakar, Senegal). In addition, the ARM-2 posture utilizes four geographically dispersed FSLs, at Eielson AFB, Alaska; Beni Suef, Egypt; Bishkek-Manas, Kyrgyzstan; and Andersen AB, Guam.

(2) Option 2: Increasing Inventory of Prepositioned Stocks. Because we had limited knowledge of the amounts of each commodity currently stored at each FSL, we assumed that there were no commodity procurement or reallocation costs in the initial NRM run and instead let the model identify the optimal allocation of commodities across FSLs. We then used this NRM run as the baseline to set initial allocation for all future runs, basing all commodity procurement and reallocation costs on variations to this baseline.

The RM posture increases the total amount of commodities positioned throughout the entire system. More specifically, there are additional commodities in the system (dispersed across several FSLs) in an amount equal to the largest aggregate allocation of commodities at any one FSL. This ensures that if the access is lost to the largest facility, there are sufficient commodities in the system to satisfy demand. For the ARM-1 posture, the model also increased the overall amount of commodities throughout the entire system. For each commodity, this increase was equal to the maximum amount of that commodity that any FOL receives from any one FSL. Note the key distinction here which explains much of the difference in procurement cost between the RM and ARM-1: the RM procures additional commodities in an amount equal to the allocation at the FSL with 
TABLE 4: Allocation of commodities across utilized FSLs.

\begin{tabular}{lccccc}
\hline Model & Minimum & Average & Maximum & Standard deviation & Coefficient of variation \\
& 1,919 & 4,743 & 9,348 & 2,672 & 0.56 \\
NRM & 2,937 & 4,437 & 5,818 & 1,063 & 0.24 \\
RM & 450 & 3,296 & 6,734 & 1,617 & 0.49 \\
ARM-1 & 1,395 & 3,474 & 5,127 & 1,137 & 0.33 \\
\hline
\end{tabular}

the largest aggregate allocation (with the FSL in question being constant for each commodity), whereas the ARM-1 procures additional commodities in an amount equal to the maximum amount of that commodity received at any FOL from any single FSL (with the FSL in question being potentially different for each commodity). A similar logic explains the additional procurement required by the ARM-2 posture, with the amount equal to the maximum amount of each commodity received at any FOL from any two FSLs. In particular, the $\mathrm{RM}$ procured an additional amount of commodities equal to 5,818 tons, whereas the ARM-1 and ARM-2 procured 8,609 and 15,099 total tons of additional commodities, respectively. Given that the ARM-2 additional procurement is nearly double that of the ARM-1 posture, we can infer that, in general, the maximum amount of each commodity sent from one FSL to one FOL is approximately equal to the amount sent from the second largest FSL-FOL pair. For example, in the validation runs for both ARM-1 and ARM-2, the FOL at Mariscal, Ecuador, receives 258 tons of munitions from its largest source, Cotopaxi, Ecuador, and an additional 258 tons of munitions from its second largest source, the APS. For ARM-1, 258 additional tons of munitions tons are procured, while for ARM-2, 516 additional tons of munitions tons are procured.

(3) Option 3: Dispersing Commodities More Evenly across FSLs. The level of dispersion of commodities among the utilized FSLs varies considerably across the four postures. Table 4 presents the minimum, average, maximum, standard deviation, and coefficient of variation ( $\mathrm{CV}$, equal to standard deviation divided by mean) of the aggregate tons of inventory stored across all utilized FSLs for each posture.

Whereas the NRM chose to place a relatively large share of the commodities at a few highly cost-effective FSLs, the reliable postures chose to disperse commodities more evenly across opened FSLs. As would be expected, the reliable postures are not as dependent on any one individual facility. Because the ARM-1 and ARM-2 postures utilize many more FSLs than do either the NRM or RM, their average values are much smaller, despite the fact that the ARM-1 and ARM-2 postures contain more total inventory. Within the three reliable postures, the ARM-1 posture was less evenly dispersed than the RM posture, with ARM-1 having a larger maximum value and smaller minimum value, along with a much larger standard deviation and CV. The ARM-2 posture has the smallest maximum allocation value across four postures, along with a level of dispersal (using
$\mathrm{CV}$ as a measure) closer to that of the RM than the other postures.

\section{Conclusions and Future Research}

The objective of this research was to develop analytic tools to assist policymakers in identifying a reliable set of facility locations for the prepositioning of rapid response materiel that can support a broad range of potential contingencies and satisfy demands even in the event of potential disruptions to the network of storage sites. This topic differs from the problems addressed in the current literature, which are generally focused on private-sector for-profit applications for which the probability of loss of access to a facility can be used to identify a network design that minimizes expected costs. Responses to humanitarian or national security demands can require a mandatory rapid response across a set of specified scenarios, without regard to probability of occurrence. This paper develops MILP models to identify alternative prepositioning postures for such an environment, where a posture is defined as a set of FSLs and an allocation of materiel across those FSLs, which meet these mandatory rapid response objectives.

As expected, the posture identified by an optimization model (NRM) that does not account for the potential loss of access to FSLs is incapable of satisfying demands in the event of loss of access. The primary weaknesses to such a posture are that it is heavily reliant on a small number of highly cost-effective FSLs and that it has no incentive to procure inventory in excess of the maximum requirements.

Accordingly, we next developed an optimization model (RM) that identifies a posture which will satisfy demand in the event of loss of access to any FSL, at minimum total cost. The posture identified by the RM is considerably more expensive than the NRM posture in the fortuitous event that no loss of access occurs to any FSL (208\% increase over the NRM posture cost), with most of this cost attributable to the RM posture's use of an Afloat Prepositioning Ship (APS). When comparing the postures, the RM made use of three strategies to build reliability into the network: it opened additional FSLs (12, versus 10 for the NRM), it increased the total amount of commodities in the system (53,244 total tons of stocks, versus 47,430 for the NRM), and it dispersed commodities more evenly across the utilized FSLs (coefficient of variation of the aggregate tons of inventory stored across all utilized FSLs equal to 0.24 , versus 0.56 for the NRM). 
However, the RM does not scale easily to consider situations in which access may be lost to multiple facilities simultaneously. Thus, we developed another model (ARM) that can be readily scaled to account for loss of access to multiple facilities. The ARM identifies a posture that is feasible against the specified loss of access scenario, but it does not identify the truly minimum-cost posture at this level of reliability, since the RM procures additional commodities in an amount equal to the allocation at the FSL with the largest aggregate allocation (with the FSL in question being constant for each commodity), whereas the ARM-1 procures additional commodities in an amount equal to the maximum amount of that commodity received at any FOL from any single FSL (with the FSL in question being potentially different for each commodity).

The ARM makes use of the same strategies for building reliability into a posture. For postures that are feasible in the event of loss of access to any single FSL (ARM-1) or to loss of access to any pair of FSLs (ARM-2): additional FSLs are utilized (17 and 18, resp.) and the total amount of commodities in the system is increased (56,032 and 62,532 total tons of stocks, resp.), but the level of dispersal of commodities across the utilized FSLs lies between those utilized by the NRM and RM (coefficients of variation of the aggregate tons of inventory stored across all utilized FSLs equal to 0.49 and 0.33 for ARM-1 and ARM-2, resp.).

In summary, this analysis found that a substantial level of reliability can be built into the prepositioning network but that this reliability incurs a relatively high increase in costs, in the event that no loss of access occurs, with costs under this scenario increasing by $206 \%$ ( $\$ 181$ million) to build a posture (RM) that is reliable in the event of loss of access to a single FSL, and a cost increase of 339\% ( $\$ 299$ million) for a posture (ARM-2) that is reliable in the event of loss of access to any two FSLs. In both cases, a large fraction of the increased cost (\$118 million) is due to the use of afloat storage.

More importantly, in the event that loss of access occurs, the nonreliable NRM posture cannot satisfy requirements at any cost, due to the short timelines required for delivery of materiel. The reliable postures not only can satisfy the delivery requirements in the event of loss of access to a storage site, but do so at minimal additional cost, with the RM posture's cost increasing by no more than $\$ 2$ million (all transportation costs) even if access is lost to a single FSL, whereas the ARM-2 posture's cost increases by no more than $\$ 6$ million (again, all transportation costs) even if access is lost to any two FSLs.

4.1. Future Research and Additional Applications. With regard to future research, for applications concerned about potential loss of access to more than two storage sites, the ARM can be easily modified to account for such situations, with no increase to the number of model variables (but a sizeable increase to the number of constraints). Such an analysis would allow the analyst to present the policymaker with a tradeoff curve showing how total costs differ with varying levels of reliability. From a methodological perspective, this research considered only disruptions to prepositioning storage sites. Future research could expand on these methods to consider potential disruptions to transportation routes (network arcs).

The reliability modeling methods we developed can be used by policymakers who need to select resource locations in the presence of uncertainty. Specifically, it should be of interest to logisticians, operators, and mobility planners throughout the Department of Defense (DoD). It should also be of interest to Homeland Security officials and industries who may be concerned about prepositioning of emergency rapid response materiel and so forth.

While this article focused on overseas basing options for the Air Force, one could also use the methods for problems in other areas including supply chains, electric power grids, telecommunications, and Homeland Security. To demonstrate how this would work, we will take an application from the disaster preparedness policy arena, the Strategic National Stockpile (SNS).

In the event of a terrorist attack or natural disaster in the United States, large quantities of medical supplies may be needed at the site of the attack or disaster. No individual can predict exactly where an attack or disaster will occur, and few state and local governments have the resources to create sufficient stockpiles on their own. Therefore, the SNS was created to provide rapid access to these medical supplies when needed. The SNS maintains large quantities of prepackaged medical supplies that are meant to supplement state and local supplies when a public health emergency occurs. The SNS has stockpiled enough medical supplies to simultaneously protect the population of several large cities.

Some examples of the supplies that are stored are medicines, vaccines, bandages, and equipment. The medical supplies, equipment, and pharmaceuticals are stored in prepacked air cargo containers, called 12-Hour Push Packages that are ready for immediate shipment. As the name implies, the 12-Hour Push Packages can be deployed anywhere in the United States within twelve hours after a request is made and the federal decision made to deploy SNS assets.

While the exact locations are not available to the general public, these medical supplies are strategically located at twelve secure warehouses near major transportation hubs in the United States. The warehouses are located in such a way as to ensure that the supplies will reach the designated site within the twelve-hour time requirement. Two critical decisions that must be made are where to locate each of the warehouses and what quantity of supplies to place at each warehouse. When making these decisions, the decision makers must take into account the possibility that, depending on the emergency, access may be lost to one or more of these warehouses. Clearly, the elements of this problem mirror the elements of the Air Force application we focused on in this article and could be solved in a similar manner.

Other applications of the model presented in this article include rapid response to natural disasters, such as earthquakes, for which current prediction abilities are limited, or intentional attacks by an adversary, such as biological attacks using smallpox, for which near-immediate response is necessary to minimize the threat to the larger population. 


\section{Appendix}

\section{FSL Site Selection and Transportation Reliability Model Formulation}

Here, we present the mathematical formulation of the reliability model (RM).

\section{Sets and Set Indices}

$i \in I$ : commodities

$j \in J:$ FSL index; $J=\{$ FSL1, FSL2, .., APF1, $\mathrm{APF} 2, \ldots\}$

$J_{1}(J) \subseteq J$ : all but first FSL

$J_{\aleph}(J) \subseteq J$ : all but last FSL

$\operatorname{AFL}(J) \subseteq J$ : afloat FSLs

$k \in K:$ FOL index; $K=\{$ FOL 1, FOL $2, \ldots\}$

$m \in M$ : modes of transport

$\operatorname{AIR}(M) \subseteq M:$ aircraft

$\mathrm{LAN}(M) \subseteq M$ : land vehicles

$\mathrm{SEA}(M) \subseteq M$ : sea vehicles

$h \in H$ : phases; $H=\{1,2, \ldots\}$

$o \in \mathrm{SCN}$ : deployment scenarios; $\mathrm{SCN}=\{1,2, \ldots\}$

$t \in T$ : time periods which divide up each phase $h$; $T=\{1,2, \ldots\}$

\section{Data Parameters}

$\Delta_{j}$ : fixed cost incurred to open FSL $j$ with $E_{j}$ square feet of storage space

$\Theta_{m h}$ : cost of obtaining an additional vehicle of mode $m$ at the beginning of phase $h$

$\Xi_{j}$ : construction cost per unit of storage needed beyond $E_{j}$ at FSL $j$

$\Upsilon_{j}$ : operating cost (discounted over the time horizon) per unit of storage at FSL $j$

$\Psi_{i k}$ : shortfall cost per time unit per ton of commodity $i$ not fulfilled at FOL $k$

$\Omega_{i j k m}$ : cost per ton of commodity $i$ transported from FSL $j$ to FOL $k$ via mode $m$

$\alpha_{m}$ : number of time periods necessary to load a mode $m$ vehicle

$\beta_{m}$ : number of time periods necessary to unload a mode $m$ vehicle

$\gamma_{m}$ : maximum load in tons per mode $m$ vehicle

$\varepsilon_{m}$ : maximum load in square feet per mode $m$ vehicle

$\zeta_{k}$ : contingency start period at FOL $k$ $\eta_{k}$ : contingency finish period at FOL $k$

$\mu_{k}$ : phase of scenario associated with FOL $k$

$\pi_{1 j m}$ : additional time needed prior to loading for commodities departing FSL $j$ via mode $m$

$\pi_{2 k m}$ : additional time needed following unloading for commodities to reach FOL $k$ via mode $m$

$\rho_{m}$ : conversion factor for parking space for mode $m$

$\sigma_{m}$ : utilization rate, expressed (for airlift) as the average flying hour goal per day divided by 24 hours, for mode $m$

$\tau_{j k m}$ : one-way transportation time from FSL $j$ to FOL

$k$ (or in opposite direction) via mode $m$

$\phi_{i}$ : conversion factor for commodity from tons to square feet of storage space

$g$ : maximum square feet per commodity $i$ unit of storage

$P_{i}$ : cost of procuring additional tons of commodity $i$

$\mathrm{IA}_{i j}$ : initial allocation (in tons) of commodity $i$ at FSL j

$L_{i}$ : cost of reallocating tons of commodity $i$ independent of FSLs

$\mathrm{STEAM}_{j k}$ : additional time needed for steaming to port before afloat FSL $j$ can begin offloading at port associated with FOL $k$

FOLSCEN $_{k}$ : scenario associated with FOL $k$

SCENPHASE $_{o}$ : phase associated with scenario $o$

$A_{\aleph j}: \max$ on ground, in class $\aleph[\operatorname{AIR}(M)=1$, $\operatorname{LAN}(M)=2, \operatorname{SEA}(M)=3$ ] equivalent vehicles, at FSL $j$

$B_{\aleph k}$ : $\max$ on ground, in class $\aleph[\operatorname{AIR}(M)=1$, $\operatorname{LAN}(M)=2, \operatorname{SEA}(M)=3$ ] equivalent vehicles, at FOL $k$

$C_{m h}$ : planned system-wide inventory of mode $m$ vehicles at the beginning of phase $h$

$D_{i k t}$ : incremental demand, in tons, for commodity $i$ at FOL $k$ at time $t$

$E_{j}$ : minimum units of storage needed for an economically feasible FSL at location $j$

$F_{j}$ : maximum potential units of storage at FSL $j$

Variables

$n n_{j}$ : additional units of storage needed beyond $E_{j}$ at FSL $j$

$q_{j m h}$ : number of mode $m$ vehicles available at FSL $j$ at the start of time period $t=1$ during phase $h$

$r_{m h}$ : additional mode $m$ vehicles obtained at the beginning of phase $h$

$s_{i k t}$ : shortfall below demand, in tons, for commodity $i$ at FOL $k$ not fulfilled by time $t$ 
$s 1_{i k t}:$ shortfall below demand, in tons, for commodity $i$ at FOL $k$ not fulfilled by time $t$ for subset $J_{1}$

$s \aleph_{i k t}$ : shortfall below demand, in tons, for commodity $i$ at FOL $k$ not fulfilled by time $t$ for subset $J_{\aleph}$

$v_{j m t h}$ : number of mode $m$ vehicles available at FSL $j$ at the end of time $t$ during phase $h$

$v 1_{j m t h}$ : number of mode $m$ vehicles available at FSL $j$ (in subset $J_{1}$ ) at the end of time $t$ during phase $h$

$v \aleph_{j m t h}$ : number of mode $m$ vehicles available at FSL $j$ (in subset $J_{\aleph}$ ) at the end of time $t$ during phase $h$

$w_{j}$ : binary variable indicating status of FSL $j$, equal to 1 if opened and equal to 0 otherwise

$x_{i j k m t}$ : tons of commodity $i$ sent from FSL $j$ to FOL $k$ via mode $m$, beginning loading on time $t$

$x 1_{i j k m t}$ : tons of commodity $i$ sent from FSL $j$ (in subset $J_{1}$ ) to FOL $k$ via mode $m$, beginning loading on time $t$

$x \aleph_{i j k m t}$ : tons of commodity $i$ sent from FSL $j$ (in subset $J_{\aleph}$ ) to FOL $k$ via mode $m$, beginning loading on time $t$

$y_{j k m t}$ : number of mode $m$ vehicles tasked to transport commodities from FSL $j$ to FOL $k$, beginning loading on time $t$. (integer).

$y 1_{j k m t}$ : number of mode $m$ vehicles tasked to transport commodities from FSL $j$ (in subset $J_{1}$ ) to FOL $k$, beginning loading on time $t$. (integer).

$y \aleph_{j k m t}$ : number of mode $m$ vehicles tasked to transport commodities from FSL $j$ (in subset $J_{\aleph}$ ) to FOL $k$, beginning loading on time $t$. (integer). $z_{j k m t}$ : number of mode $m$ vehicles tasked to make the return trip from FOL $k$ to FSL $j$, departing on time $t$. (integer).

$z 1_{j k m t}$ : number of mode $m$ vehicles tasked to make the return trip from FOL $k$ to FSL $j$ (in subset $J_{1}$ ), departing on time $t$. (integer).

$z \aleph_{j k m t}$ : number of mode $m$ vehicles tasked to make the return trip from FOL $k$ to FSL $j$ (in subset $J_{N}$ ), departing on time $t$. (integer).

$w w_{j}$ : units of storage utilized at FSL $j$

$x x_{i j}$ : maximum total tons of commodity $i$ sent out of FSL $j$ across the full set of FSLs and all $\aleph$ subsets of FSLs

$p p_{j h o}$ : binary variable indicating if afloat FSL $j$ supports scenario $o$ during phase $h$

$\mathrm{AU}_{i j}$ : additional units of commodity $i$ (in tons) that is procured at FSL $j$ beyond the initial amount $\left(\mathrm{IA}_{i j}\right)$, this is a gain of inventory at FSL $j$

$\mathrm{EI}_{i j}$ : inventory of commodity $i$ (in tons) at FSL $j$ that is reallocated to other FSLs, this is a loss of inventory at FSL $j$

$\mathrm{AP}_{i}$ : the sum of the additional amount (in tons) of commodity $i$ procured across the entire system, this is a gain of inventory across all FSLs

$b_{i j}$ : binary variable, equal to 1 if FSL $j$ reallocates commodity $i$ to other FSLs and equal to 0 otherwise

$m$ : a very large positive number to ensure that FSL $j$ can reallocate commodity $i$ to other FSLs or procure additional units of commodity $i$ but not both

Note. There is an implicit assumption throughout the entire model that terms having an index value $t \leq 0$ are not considered.

Objective Function

$$
\begin{aligned}
\min & \sum_{j}\left(\Delta_{j} w_{j}+\Xi_{j} n n_{j}+\Upsilon_{j} w w_{j}\right)+\left[\sum_{i j k m t} \frac{\Omega_{i j k m}}{\aleph+1} x_{i j k m t}+\sum_{i k m t} \sum_{j \in J_{1}(J)} \frac{\Omega_{i j k m}}{\aleph+1} x 1_{i j k m t}+\cdots+\sum_{i k m t} \sum_{j \in J_{\aleph}(J)} \frac{\Omega_{i j k m}}{\aleph+1} x \aleph_{i j k m t}\right] \\
& +\sum_{m h} \Theta_{m h} r_{m h}+\sum_{i} P_{i} \mathrm{AP}_{i}+\sum_{i j} L_{i} \mathrm{EI}_{i j}+\left[\sum_{i k t} \frac{\Psi_{i k}}{\aleph+1} s_{i k t}+\sum_{i k t} \frac{\Psi_{i k}}{\aleph+1} s 1_{i k t}+\cdots+\sum_{i k t} \frac{\Psi_{i k}}{\aleph+1} s \aleph_{i k t}\right] .
\end{aligned}
$$

The objective function minimizes the average total cost, equal to the sum of the FSL opening cost, the cost for construction of new facilities, the facility operation cost, the average transport cost across all scenarios, the cost of procuring new vehicles, the cost for reallocating commodities between FSLs, the cost of procuring additional commodities, and the average shortfall cost across all scenarios for not satisfying demand requirements. 


\section{Constraints}

$$
\begin{aligned}
& \sum_{j} q_{j m h} \leq\left(C_{m h}+r_{m h}\right) \quad \forall m, h, \\
& \sum_{k \ni \mu_{k}=h} y_{j k m t} \leq v_{j m(t-1) h} \quad \forall j, m, h ; t \geq 2, \\
& \sum_{k \ni \mu_{k}=h} y 1_{j k m t} \leq v 1_{j m(t-1) h} \quad \forall m, h ; j \in J_{1}(J), t \geq 2, \\
& \vdots \\
& \sum_{k \ni \mu_{k}=h} y \aleph_{j k m t} \leq v \aleph_{j m(t-1) h} \quad \forall m, h ; j \in J_{\mathcal{N}}(J), t \geq 2, \\
& \sum_{k \ni \mu_{k}=h} \sum_{m \in \operatorname{AIR}(M)} \sum_{n=0}^{\alpha_{m}-1}\left[\rho_{m}\left(y_{j k m(t-n)}\right)\right] \leq A{ }^{\prime \prime}{ }^{\prime \prime j} \quad \forall j, t, h, \\
& \sum_{k \ni \mu_{k}=h} \sum_{m \in \operatorname{AIR}(M)} \sum_{n=0}^{\alpha_{m}-1}\left[\rho_{m}\left(y 1_{j k m(t-n)}\right)\right] \leq A^{\prime \prime 1 " j}
\end{aligned}
$$

$\forall t, h ; j \in J_{1}(J)$,

$$
\sum_{k \ni \mu_{k}=h} \sum_{m \in \operatorname{AIR}(M)} \sum_{n=0}^{\alpha_{m}-1}\left[\rho_{m}\left(y \aleph_{j k m(t-n)}\right)\right] \leq A{ }_{1 " j}
$$

$\forall t, h ; j \in J_{\mathbb{N}}(J)$,

$\sum_{k \ni \mu_{k}=h} \sum_{m \in \operatorname{LAN}(M)} \sum_{n=0}^{\alpha_{m}-1}\left[\rho_{m}\left(y_{j k m(t-n)}\right)\right] \leq A_{“ 2 " j} \quad \forall j, t, h$,

$$
\sum_{k \ni \mu_{k}=h} \sum_{m \in \operatorname{LAN}(M)} \sum_{n=0}^{\alpha_{m}-1}\left[\rho_{m}\left(y 1_{j k m(t-n)}\right)\right] \leq A_{{ }^{2}{ }^{\prime} j}
$$

$\forall t, h ; j \in J_{1}(J)$,

$$
\begin{aligned}
& \sum_{k \ni \mu_{k}=h} \sum_{m \in \operatorname{LAN}(M)} \sum_{n=0}^{\alpha_{m}-1}\left[\rho_{m}\left(y \aleph_{j k m(t-n)}\right)\right] \leq A_{{ }^{2} " j} \\
& \sum_{k \ni \mu_{k}=h} \sum_{m \in \operatorname{SEA}(M)} \sum_{n=0}^{\alpha_{m}-1}\left[\rho_{m}\left(y 1_{j k m(t-n)}\right)\right] \leq A{ }^{\prime \prime}{ }^{\prime}
\end{aligned}
$$

$$
\sum_{k \ni \mu_{k}=h} \sum_{m \in \operatorname{SEA}(M)} \sum_{n=0}^{\alpha_{m}-1}\left[\rho_{m}\left(y \aleph_{j k m(t-n)}\right)\right] \leq A_{“ 3^{\prime} j}
$$

$\forall t, h ; j \in J_{\mathbb{N}}(J)$,

$$
\sum_{j} \sum_{m \in \operatorname{AIR}(M)} \sum_{n=0}^{\beta_{m}-1}\left[\rho_{m}\left(y_{j k m\left(t-\tau_{j k m}-\alpha_{m}-n\right)}\right)\right] \leq B{ }^{\prime \prime} k
$$

$\forall k ; \zeta_{k} \leq t \leq \eta_{k}$

$$
\sum_{j \in J_{1}(J)} \sum_{m \in \operatorname{AIR}(M)} \sum_{n=0}^{\beta_{m}-1}\left[\rho_{m}\left(y 1_{j k m\left(t-\tau_{j k m}-\alpha_{m}-n\right)}\right)\right] \leq B{ }{ }^{\prime \prime k}
$$

$\forall k ; \zeta_{k} \leq t \leq \eta_{k}$

$$
\sum_{j \in J_{\mathbb{N}}(J)} \sum_{m \in \operatorname{AIR}(M)} \sum_{n=0}^{\beta_{m}-1}\left[\rho_{m}\left(y \aleph_{j k m\left(t-\tau_{j k m}-\alpha_{m}-n\right)}\right)\right] \leq B{ } " k
$$

$\forall k ; \zeta_{k} \leq t \leq \eta_{k}$,

$$
\sum_{j} \sum_{m \in \operatorname{LAN}(M)} \sum_{n=0}^{\beta_{m}-1}\left[\rho_{m}\left(y_{j k m\left(t-\tau_{j k m}-\alpha_{m}-n\right)}\right)\right] \leq B \text { “2" } " k
$$

$\forall k ; \zeta_{k} \leq t \leq \eta_{k}$

$$
\sum_{j \in J_{1}(J)} \sum_{m \in \operatorname{LAN}(M)} \sum_{n=0}^{\beta_{m}-1}\left[\rho_{m}\left(y 1_{j k m\left(t-\tau_{j k m}-\alpha_{m}-n\right)}\right)\right] \leq B \text { “" } " k
$$

$\forall k ; \zeta_{k} \leq t \leq \eta_{k}$

$\sum_{j \in J_{\mathbb{N}}(J)} \sum_{m \in \operatorname{LAN}(M)} \sum_{n=0}^{\beta_{m}-1}\left[\rho_{m}\left(y \aleph_{j k m\left(t-\tau_{j k m}-\alpha_{m}-n\right)}\right)\right] \leq B{ } " k$

$\forall k ; \zeta_{k} \leq t \leq \eta_{k}$

$\sum_{j} \sum_{m \in \operatorname{SEA}(M)} \sum_{n=0}^{\beta_{m}-1}\left[\rho_{m}\left(y_{j k m\left(t-\tau_{j k m}-\alpha_{m}-n\right)}\right)\right] \leq B{ }^{\prime \prime k}$

$\forall k ; \zeta_{k} \leq t \leq \eta_{k}$

$\sum_{j \in J_{1}(J)} \sum_{m \in \operatorname{SEA}(M)} \sum_{n=0}^{\beta_{m}-1}\left[\rho_{m}\left(y 1_{j k m\left(t-\tau_{j k m}-\alpha_{m}-n\right)}\right)\right] \leq B 3^{\prime \prime k}$

$\forall k ; \zeta_{k} \leq t \leq \eta_{k}$,

$$
\sum_{j \in J_{\mathbb{N}}(J)} \sum_{m \in \operatorname{SEA}(M)} \sum_{n=0}^{\beta_{m}-1}\left[\rho_{m}\left(y \aleph_{j k m\left(t-\tau_{j k m}-\alpha_{m}-n\right)}\right)\right] \leq B{ }^{\prime \prime}{ }^{\prime \prime} k
$$

$\forall k ; \zeta_{k} \leq t \leq \eta_{k}$

$\sum_{j, m} \sum_{n=1}^{t} x_{i j k m\left(n-\tau_{j k m}-\alpha_{m}-\beta_{m}-\pi_{2 k m}\right)} \geq\left(\sum_{n=1}^{t} D_{i k n}\right)-s_{i k t}$

$\forall i, k ; \zeta_{k} \leq t \leq \eta_{k}$,

$\sum_{m} \sum_{j \in J_{1}(J)} \sum_{n=1}^{t} x 1_{i j k m\left(n-\tau_{j k m}-\alpha_{m}-\beta_{m}-\pi_{2 k m}\right)} \geq\left(\sum_{n=1}^{t} D_{i k n}\right)-s 1_{i k t}$

$\forall i, k ; \zeta_{k} \leq t \leq \eta_{k}$,

$\sum_{m} \sum_{j \in J_{\mathbb{N}}(J)} \sum_{n=1}^{t} x \aleph_{i j k m\left(n-\tau_{j k m}-\alpha_{m}-\beta_{m}-\pi_{2 k m}\right)} \geq\left(\sum_{n=1}^{t} D_{i k n}\right)-s \aleph_{i k t}$ $\forall i, k ; \zeta_{k} \leq t \leq \eta_{k}$,

$\sum_{i} \phi_{i} x x_{i j} \leq g\left(E_{j} w_{j}+n n_{j}\right) \quad \forall j$,

$\sum_{i} \phi_{i} x x_{i j} \leq g\left(w w_{j}\right) \quad \forall j$, 


$$
\begin{aligned}
& n n_{j} \leq\left(F_{j}-E_{j}\right) w_{j} \quad \forall j, \\
& \sum_{k \ni \mu_{k}=h} \sum_{j}\left(\left[\sum_{t} \tau_{j k m}\left(y_{j k m t}\right)\right]+\left[\sum_{t=1}^{|T|-\tau_{j k m}} \tau_{j k m} z_{j k m t}\right]\right. \\
& \left.+\left[\sum_{t=|T|-\tau_{j k m}+1}^{|T|-1}(|T|-t) z_{j k m t}\right]\right) \leq|T|\left(C_{m h}+r_{m h}\right) \sigma_{m} \\
& \sum_{k \ni \mu_{k}=h} \sum_{j \in J_{1}(J)}\left(\left[\sum_{t} \tau_{j k m}\left(y 1_{j k m t}\right)\right]+\left[\sum_{t=1}^{|T|-\tau_{j k m}} \tau_{j k m} z 1_{j k m t}\right]\right. \\
& \left.+\left[\sum_{t=|T|-\tau_{j k m}+1}^{|T|-1}(|T|-t) z 1_{j k m t}\right]\right) \leq|T|\left(C_{m h}+r_{m h}\right) \sigma_{m} \\
& \sum_{k \ni \mu_{k}=h} \sum_{j \in J_{\mathbb{N}}(J)}\left(\left[\sum_{t} \tau_{j k m}\left(y \aleph_{j k m t}\right)\right]+\left[\sum_{t=1}^{|T|-\tau_{j k m}} \tau_{j k m} z \aleph_{j k m t}\right]\right. \\
& \left.+\left[\sum_{t=|T|-\tau_{j k m}+1}^{|T|-1}(|T|-t) z \aleph_{j k m t}\right]\right) \leq|T|\left(C_{m h}+r_{m h}\right) \sigma_{m}
\end{aligned}
$$

$\forall m, h$,

$\sum_{i} x_{i j k m t} \leq \gamma_{m} y_{j k m t}$

$$
\forall j, k, m, \zeta_{k}+\pi_{1 j m} \leq t \leq \eta_{k}-\tau_{j k m}-\alpha_{m}-\beta_{m}-\pi_{2 k m},
$$

$\sum_{i} x 1_{i j k m t} \leq \gamma_{m} y 1_{j k m t}$

$\forall k, m ; j \in J_{1}(J), \zeta_{k}+\pi_{1 j m} \leq t \leq \eta_{k}-\tau_{j k m}-\alpha_{m}-\beta_{m}-\pi_{2 k m}$,

$\sum_{i} x \aleph_{i j k m t} \leq \gamma_{m} y \aleph_{j k m t}$

$\forall k, m ; j \in J_{\mathbb{N}}(J), \zeta_{k}+\pi_{1 j m} \leq t \leq \eta_{k}-\tau_{j k m}-\alpha_{m}-\beta_{m}-\pi_{2 k m}$,

$\sum_{i} \phi_{i} x_{i j k m t} \leq \varepsilon_{m} y_{j k m t}$

$$
\forall j, k, m, \zeta_{k}+\pi_{1 j m} \leq t \leq \eta_{k}-\tau_{j k m}-\alpha_{m}-\beta_{m}-\pi_{2 k m}
$$

$\sum_{i} \phi_{i} x 1_{i j k m t} \leq \varepsilon_{m} y 1_{j k m t}$

$\forall k, m ; j \in J_{1}(J), \zeta_{k}+\pi_{1 j m} \leq t \leq \eta_{k}-\tau_{j k m}-\alpha_{m}-\beta_{m}-\pi_{2 k m}$,

$\sum_{i} \phi_{i} x \aleph_{i j k m t} \leq \varepsilon_{m} y \aleph_{j k m t}$

$\forall k, m ; j \in J_{N}(J), \zeta_{k}+\pi_{1 j m} \leq t \leq \eta_{k}-\tau_{j k m}-\alpha_{m}-\beta_{m}-\pi_{2 k m}$,

$$
\sum_{j} z_{j k m t}=\sum_{j} y_{j k m\left(t-\tau_{j k m}-\alpha_{m}-\beta_{m}\right)}
$$

$\forall k, m, \zeta_{k}+\alpha_{m}+\beta_{m} \leq t \leq \eta_{k}-\pi_{2 k m}$

$$
\sum_{j \in J_{1}(J)} z 1_{j k m t}=\sum_{j \in J_{1}(J)}\left[y 1_{j k m\left(t-\tau_{j k m}-\alpha_{m}-\beta_{m}\right)}\right]
$$

$\forall k, m, \zeta_{k}+\alpha_{m}+\beta_{m} \leq t \leq \eta_{k}-\pi_{2 k m}$

$$
\sum_{j \in J_{\mathbb{N}}(J)} z \aleph_{j k m t}=\sum_{j \in J_{\mathbb{N}}(J)}\left[y \aleph_{j k m\left(t-\tau_{j k m}-\alpha_{m}-\beta_{m}\right)}\right]
$$

$\forall k, m, \zeta_{k}+\alpha_{m}+\beta_{m} \leq t \leq \eta_{k}-\pi_{2 k m}$

$v_{j m t h}=v_{j m(t-1) h}+\sum_{k \ni \mu_{k}=h}\left[z_{j k m\left(t-\tau_{j k m}\right)}-y_{j k m t}\right] \quad \forall j, m, h ; t \geq 2$,

$v 1_{j m t h}=v 1_{j m(t-1) h}+\sum_{k \ni \mu_{k}=h}\left[z 1_{j k m\left(t-\tau_{j k m}\right)}-y 1_{j k m t}\right]$

$\forall m, h ; j \in J_{1}(J), t \geq 2$,

$$
\begin{array}{r}
v \aleph_{j m t h}=v \aleph_{j m(t-1) h}+\sum_{k \ni \mu_{k}=h}\left[z \aleph_{j k m\left(t-\tau_{j k m}\right)}-y \aleph_{j k m t}\right] \\
\forall m, h ; j \in J_{\aleph}(J), t \geq 2,
\end{array}
$$

$v_{j m^{\prime \prime} 1 " h}=q_{j m h}-\sum_{k \ni \mu_{k}=h} y_{j k m^{* 1}} \quad \forall j, m, h$,

$v 1_{j m^{\prime \prime} 1^{\prime \prime} h}=q_{j m h}+\sum_{k \ni \mu_{k}=h}\left[-y 1_{j k m^{\prime \prime} 1^{\prime \prime}}\right] \quad \forall m, h ; j \in J_{1}(J)$,

$v \aleph_{j m^{\prime \prime} 1 " h}=q_{j m h}+\sum_{k \ni \mu_{k}=h}\left[-y \aleph_{j k m}{ }^{\prime \prime} 1 "\right] \quad \forall m, h ; j \in J_{\mathcal{N}}(J)$,

$x x_{i j}+\mathrm{EI}_{i j} \leq \mathrm{IA}_{i j}+\mathrm{AU}_{i j} \quad \forall i, j$,

$\sum_{j} \mathrm{AU}_{i j} \leq \sum_{j} \mathrm{EI}_{i j}+\mathrm{AP}_{i} \quad \forall i$

$\mathrm{EI}_{i j} \leq b_{i j} m \quad \forall i, j$,

$\mathrm{AU}_{i j} \leq\left(1-b_{i j}\right) m \quad \forall i, j$,

$\sum_{o} p p_{j h o} \leq 1 \quad \forall h ; j \in \operatorname{AFL}(J)$,

$\sum_{i m t} \sum_{k \ni \mathrm{FOLSCN}_{k}=o} x_{i j k m t} \leq\left(\sum_{i k t} D_{i k t}\right) \sum_{h=\mathrm{SCENPHASE}_{o}} p p_{j h o}$

$\forall o ; j \in \operatorname{AFL}(J)$,

$\sum_{i m t} \sum_{k \ni \operatorname{FOLSCEN}_{k}=o} x 1_{i j k m t} \leq\left(\sum_{i k t} D_{i k t}\right) \sum_{h=\operatorname{SCENPHASE}_{o}} p p_{j h o}$

$\forall o ; j \in \operatorname{AFL}\left(J_{1}\right)$,

$\sum_{i m t} \sum_{k \ni \text { FOLSCEN }_{k}=o} x \aleph_{i j k m t} \leq\left(\sum_{i k t} D_{i k t}\right) \sum_{h=\text { SCENPHASE }_{o}} p p_{j h o}$

$\forall o ; j \in \operatorname{AFL}\left(J_{\aleph}\right)$,

$\sum_{k m t} x_{i j k m t} \leq x x_{i j} \quad \forall i, j$, 


$$
\begin{gathered}
\sum_{k m t} x 1_{i j k m t} \leq x x_{i j} \quad \forall i ; j \in J_{1}(J), \\
\vdots \\
\sum_{k m t} x \aleph_{i j k m t} \leq x x_{i j} \quad \forall i ; j \in J_{\aleph}(J), \\
x_{i j k m t}=0 \quad t<\zeta_{k}+\pi_{1 j m} ; t>\eta_{k}-\tau_{j k m}-\alpha_{m}-\beta_{m}-\pi_{2 k m}, \\
x 1_{i j k m t}=0 \quad t<\zeta_{k}+\pi_{1 j m} ; t>\eta_{k}-\tau_{j k m}-\alpha_{m}-\beta_{m}-\pi_{2 k m}, \\
\vdots \\
x \aleph_{i j k m t}=0 \quad t<\zeta_{k}+\pi_{1 j m} ; t>\eta_{k}-\tau_{j k m}-\alpha_{m}-\beta_{m}-\pi_{2 k m}, \\
x_{i j k m t}, x 1_{i j k m t}, \ldots, x \aleph_{i j k m t}, x x_{i j}, s_{i k t}, s 1_{i k t}, \ldots, s \aleph_{i k t}, s s_{i k}, y_{j k m t}, \\
y 1_{j k m t}, \ldots, y \aleph_{j k m t}, z_{j k m t}, z 1_{j k m t}, \ldots, z \aleph_{j k m t}, v_{j m t h}, v 1_{j m t h}, \ldots, \\
v \aleph_{j m t h}, n n_{j}, q_{j m h}, r_{m h}, w w_{j}, \mathrm{AU}_{i j}, \mathrm{EI}_{i j}, \mathrm{AP}_{i} \geq 0, \\
y_{j k m t}, y 1_{j k m t}, \ldots, y \aleph_{j k m t}, z_{j k m t}, z 1_{j k m t}, \ldots, z \aleph_{j k m t} \text { integer } \\
w_{j}, p p_{j h o}, b \in\{0,1\} .
\end{gathered}
$$

\section{Competing Interests}

The authors declare that they have no competing interests.

\section{Acknowledgments}

RAND Project Air Force provided financial support for this research, which was performed as part of the first author's Ph.D. dissertation [24] while being a student at the Pardee RAND Graduate School.

\section{References}

[1] G. Pricks, "Report: Kyrgyzstan to close key U.S. Base," CNN.com, 2009, http://www.cnn.com/2009/WORLD/asiapcf/ 02/03/kyrgyz.base/index.html?iref=newssearch.

[2] L. V. Snyder, Supply chain robustness and reliability: models and algorithms [Ph.D. thesis], 2003, http://www.lehigh.edu/ lvs2/ Papers/LVSnyder_dissertation.pdf.

[3] M. S. Daskin, L. V. Snyder, and R. T. Berger, "Facility location in supply chain design," in Logistics Systems: Design and Optimization, A. Langevin and D. Riopel, Eds., chapter 2, pp. 39-65, Springer, New York, NY, USA, 2005.

[4] M. Caserta and S. Voß, "An exact algorithm for the reliability redundancy allocation problem," European Journal of Operational Research, vol. 244, no. 1, pp. 110-116, 2015.

[5] M. L. Schooman, Reliability of Computer Systems and Networks: Fault Tolerance, Analysis, and Design, John Wiley and Sons, New York, NY, USA, 2002.

[6] C. Lemaréchal, A. Ouorou, and G. Petrou, "Robust network design in telecommunications under polytope demand uncertainty, European Journal of Operational Research, vol. 206, no. 3, pp. 634-641, 2010.

[7] R. Church and C. ReVelle, "The maximal covering location problem," Papers of the Regional Science Association, vol. 32, no. 1, pp. 101-118, 1974.
[8] L. V. Snyder and M. S. Daskin, "Reliability models for facility location: the expected failure cost case," Transportation Science, vol. 39, no. 3, pp. 400-416, 2005.

[9] Y. Meepetchdee and N. Shah, "Logistical network design with robustness and complexity considerations," International Journal of Physical Distribution and Logistics Management, vol. 37, no. 3, pp. 201-222, 2007.

[10] B. Hamaide, H. J. Albers, and G. Busby, "Backup coverage models in nature reserve site selection with spatial spread risk heterogeneity," Socio-Economic Planning Sciences, vol. 48, no. 2, pp. 158-167, 2014.

[11] T. Cui, Y. Ouyang, and Z.-J. M. Shen, "Reliable facility location design under the risk of disruptions," Operations Research, vol. 58, no. 4, part 1, pp. 998-1011, 2010.

[12] Z.-J. M. Shen, R. L. Zhan, and J. Zhang, "The reliable facility location problem: formulations, heuristics, and approximation algorithms," INFORMS Journal on Computing, vol. 23, no. 3, pp. 470-482, 2011.

[13] L. V. Snyder, Z. Atan, P. Peng, Y. Rong, A. J. Schmitt, and B. Sinsoysal, "OR/MS models for supply chain disruptions: a review," IIE Transactions, vol. 48, no. 2, pp. 89-109, 2016.

[14] D. P. Morton, J. Salmeron, and R. K. Wood, "A stochastic program for optimizing military sealift subject attack," Military Operations Research, vol. 14, no. 2, pp. 19-39, 2009.

[15] J. M. Mulvey, R. J. Vanderbei, and S. A. Zenios, "Robust optimization of large-scale systems," Operations Research, vol. 43, no. 2, pp. 264-281, 1995.

[16] L. V. Snyder, M. P. Scaparra, M. S. Daskin, and R. L. Church, "Planning for disruptions in supply chain networks," in INFORMS Tutorials in Operations Research, pp. 234-257, INFORMS, 2006.

[17] J. Salmeron, K. Wood, and R. Baldick, "Analysis of electric grid security under terrorist threat," IEEE Transactions on Power Systems, vol. 19, no. 2, pp. 905-912, 2004.

[18] G. Brown, M. Carlyle, J. Salmerón, and K. Wood, "Defending critical infrastructure," Interfaces, vol. 36, no. 6, pp. 530-544, 2006.

[19] M. A. Amouzegar, R. G. McGarvey, R. S. Tripp, L. Luangkesorn, T. E. Lang, and C. R. Roll Jr., Evaluation of Options for Overseas Combat Support Basing, RAND Corporation, Santa Monica, Calif, USA, 2006.

[20] R. G. McGarvey, R. S. Tripp, R. Rue et al., Global Combat Support Basing: Robust Prepositioning Strategies for Air Force War Reserve Materiel, RAND Corporation, Santa Monica, Calif, USA, 2010.

[21] G. Galindo and R. Batta, "Prepositioning of supplies in preparation for a hurricane under potential destruction of prepositioned supplies," Socio-Economic Planning Sciences, vol. 47, no. 1, pp. 20-37, 2013.

[22] D. Snyder and P. Mills, Supporting Air and Space Expeditionary Forces: A Methodology for Determining Air Force Deployment Requirements, RAND Corporation, Santa Monica, Calif, USA, 2004.

[23] United States Air Force, Air Mobility Planning Factors, AFPAM 10-1403, U.S. Air Force, Washington, DC, USA, 2003.

[24] E. Lang Thomas, Defining and Evaluating Reliable Options for Overseas Combat Support Basing, Pardee RAND Graduate School Dissertation RGSD-250, 2009. 


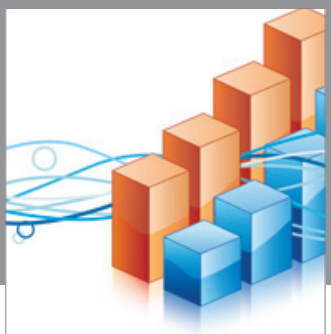

Advances in

Operations Research

vatem alat4

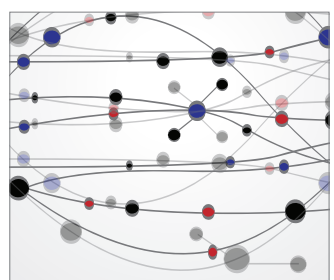

\section{The Scientific} World Journal
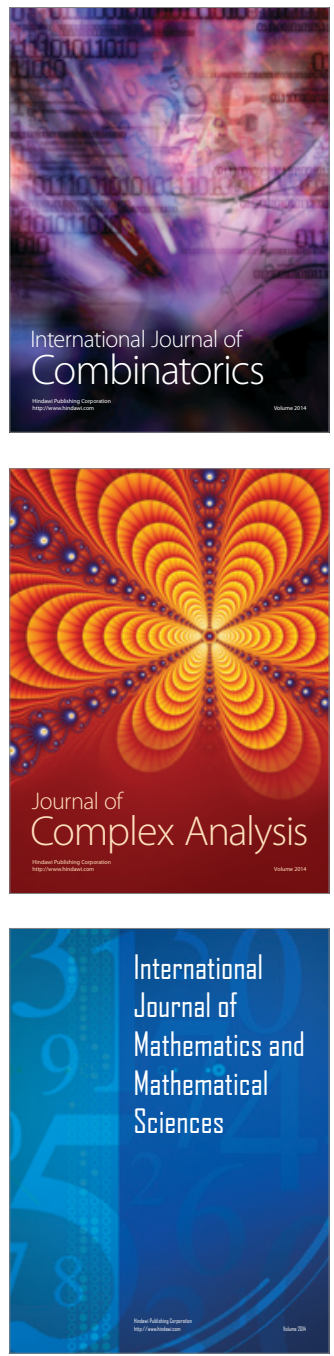
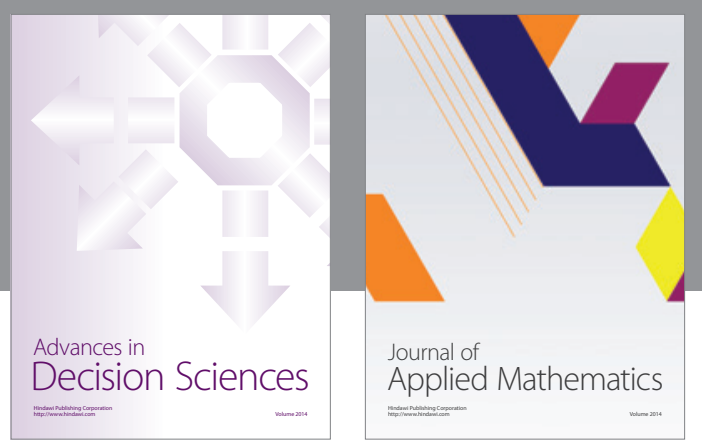

Algebra

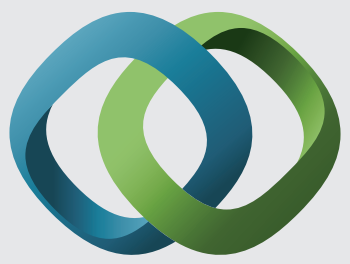

\section{Hindawi}

Submit your manuscripts at

http://www.hindawi.com
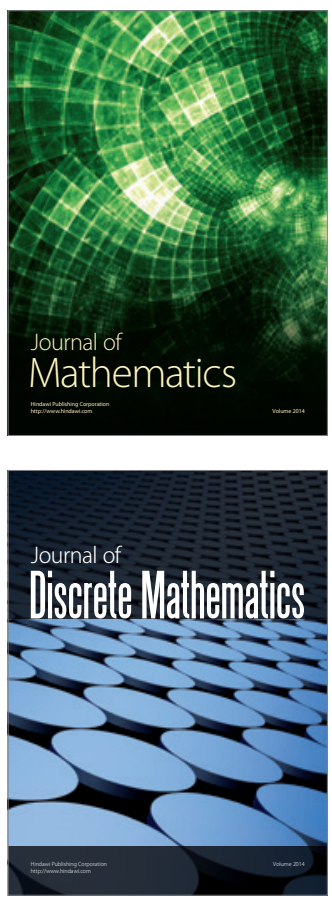

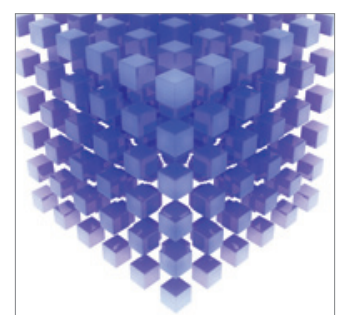

Mathematical Problems in Engineering
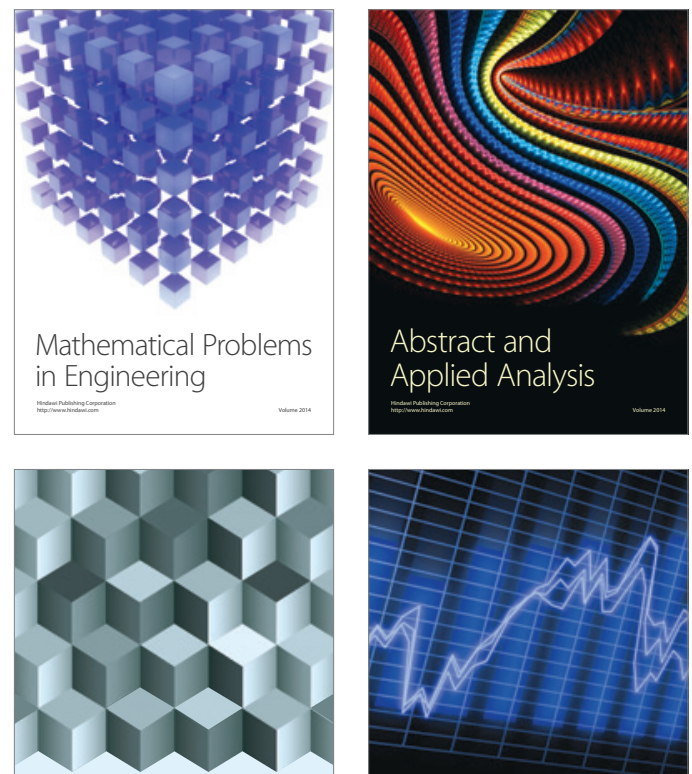

Journal of

Function Spaces

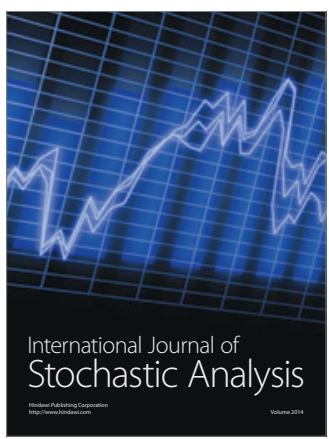

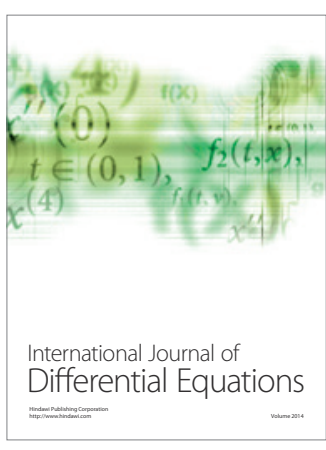
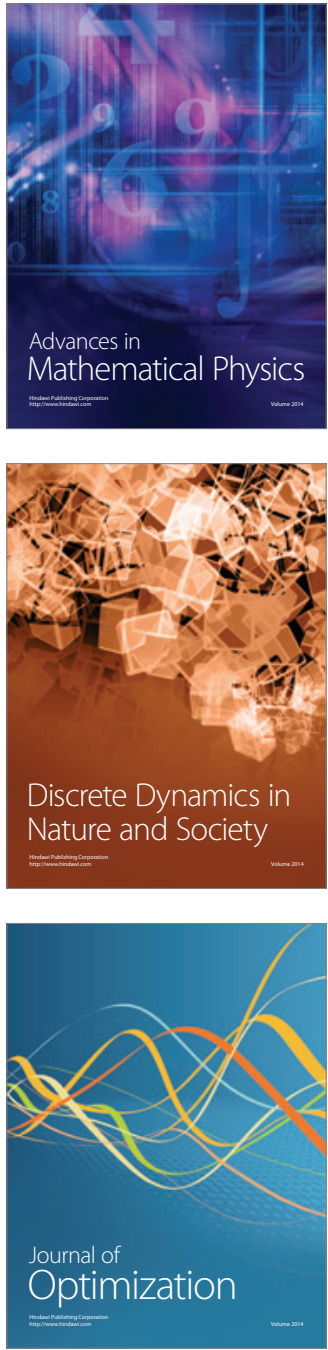